\title{
Bifurcation Analysis and Chaos Control in a Discrete-Time Parasite-Host Model
}

\author{
Xueli Chen ${ }^{1,2}$ and Lishun Ren ${ }^{1}$ \\ ${ }^{1}$ Department of Mathematics and Statistics, Zhoukou Normal University, Zhoukou, Henan 466000, China \\ ${ }^{2}$ Department of Mathematics and Statistics, Zhengzhou University, Zhengzhou, Henan 450001, China
}

Correspondence should be addressed to Lishun Ren; 9236rls@sina.com

Received 25 August 2016; Revised 30 October 2016; Accepted 29 November 2016; Published 23 January 2017

Academic Editor: Hassan A. El-Morshedy

Copyright ( 2017 Xueli Chen and Lishun Ren. This is an open access article distributed under the Creative Commons Attribution License, which permits unrestricted use, distribution, and reproduction in any medium, provided the original work is properly cited.

A discrete-time parasite-host system with bifurcation is investigated in detail in this paper. The existence and stability of nonnegative fixed points are explored and the conditions for the existence of flip bifurcation and Neimark-Sacker bifurcation are derived by using the center manifold theorem and bifurcation theory. And we also prove the chaos in the sense of Marotto. The numerical simulations not only illustrate the consistence with the theoretical analysis, but also exhibit other complex dynamical behaviors, such as bifurcation diagrams, Maximum Lyapunov exponents, and phase portraits. More specifically, when the integral step size is chosen as a bifurcation parameter, this paper presents the finding of period orbits, attracting invariant cycles and chaotic attractors of the discrete-time parasite-host system. Specifically, we have stabilized the chaotic orbits at an unstable fixed point by using the feedback control method.

\section{Introduction}

In the theory of ecology, population dynamics are generally governed by continuous-time and discrete-time systems. In recent years, more and more attention is being paid to discrete-time population models [1-20]. We can get more accurate numerical simulation results and richer dynamical behaviors from discrete-time models. In addition, many scholars are interested in how to discretize continuous population dynamical models and study their dynamical properties [21-24]. The recent years, a number of articles which investigated the flip bifurcation, fold bifurcation, pitchfork bifurcation and in the sense of Marotto's chaos of the discrete system were present in [25-29].

We investigate the discrete-time parasite-host system in this paper. In some cases, parasites can reduce host density and induce host population extinction. Ebert et al. [30] formulated the following epidemiological microparasite model:

$$
\begin{aligned}
& \dot{x}(t)=a(x+\theta y)\left(1-\frac{x+y}{K}\right)-d x-\beta x y, \\
& \dot{y}(t)=\beta x y-(d+\alpha) y,
\end{aligned}
$$

to understand how six microparasites regulate Daphnia populations and drive the populations to extinction, where $x(t)$ and $y(t)$ denote uninfected (susceptible) and infected (infected) hosts densities at time $t$, respectively; $a$ is the maximum per capita birth rate of uninfected hosts; $\theta(0 \leqslant$ $\theta \leqslant 1)$ is the relative fecundity of an infected host; $K$ is the carrying capacity of for the host population; $d$ is the parasiteindependent host background mortality; $\beta$ is the constant infection rate and $\alpha$ is the parasite-induced excess death rate; all parameters are positive.

In this model, the microparasite transmission is assumed via a mass action process, the fecundity of uninfected host is density-dependent, and the fecundity of infected host may be reduced due to being infected compared with that of uninfected host. Here, $0 \leq \theta \leq 1$ means that the fecundity of infected hosts is reduced, but they still have certain fecundity, $\theta=0$ means that infected hosts completely lose fecundity; $\theta=1$ means that the fecundity of infected hosts is not affected by parasite infection. For model (1), when $a>d$, there is always equilibrium $(0,0)$ which is a saddle. This implies that extinction of host is impossible when $a>d$, that is to say, when $a>d$ host always persists. But, in 
this model, the simulation for the stochastic model indicates that extinction of host likely occurs in certain parameter regions.

Rescaling system (1) by the following as [21, 31],

$$
\begin{aligned}
& \bar{x}=\frac{x}{K}, \\
& \bar{y}=\frac{y}{K}, \\
& \bar{t}=a t
\end{aligned}
$$

and removing the bars, then system (1) becomes in the following form:

$$
\begin{aligned}
& \dot{x}(t)=(x+\theta y)(1-x-y)-\delta x-s x y, \\
& \dot{y}(t)=s x y-(\delta+\gamma) y,
\end{aligned}
$$

where $s=\beta K / a, \delta=d / a$, and $\gamma=\alpha / a$ are positive constants. Applying the forward Euler discrete scheme to system (3), we obtain the following discrete-time parasite-host system as follows:

$$
\begin{aligned}
F:\left(\begin{array}{l}
x \\
y
\end{array}\right) & \\
& \longrightarrow\left(\begin{array}{c}
x+h[(x+\theta y)(1-x-y)-\delta x-s x y] \\
y+h[s x y-(\delta+\gamma) y]
\end{array}\right),
\end{aligned}
$$

where $h$ is the integral step size. The fixed points of system (4) satisfy the following equations:

$$
\begin{array}{r}
x+h[(x+\theta y)(1-x-y)-\delta x-s x y]=x, \\
y+h[s x y-(\delta+\gamma) y]=y,
\end{array}
$$

and then

$$
\begin{array}{r}
(x+\theta y)(1-x-y)-\delta x-s x y=0, \\
s x y-(\delta+\gamma) y=0 .
\end{array}
$$

In our paper, we apply the forward Euler Scheme to discrete the parasite-past model and mainly focus on the existence and stability of nonnegative fixed points and flip bifurcation, Neimark-Sacker bifurcation, and possible chaos in the sense of Marotto's definition [32] in the discrete-time parasite-host system by using the center manifold theorem [33] and the bifurcation theory [17-20, 33-36]. When the integral step size $h$ is taken as a bifurcation parameter, the detailed existence conditions of flip bifurcation and NeimarkSacker bifurcation are given in a very strict mathematical way. Numerical simulations are shown, including maximum Lyapunov exponents, bifurcation diagrams, and phase portraits, to verify theoretical analyses, and display some new and interesting nonlinear dynamical behaviors of the parasite-host system. In particular, one of our investigations demonstrates that the integral step size makes difference with respect to nonlinear dynamical behaviors of the discrete parasite-host system when the integral step size is taken into account as a bifurcation parameter.

The organization of this paper is as follows. In Section 2, the theorem on the existence and stability of fixed points for system (4) is proved. In Section 3, we give the sufficient conditions of existence for flip bifurcation and NeimarkSacker bifurcation by using the center manifold theorem and bifurcation theory. In Section 4, we first rigorously prove the existence of chaos in the sense of Marotto's definition. Numerical simulations are presented not only to verify the theoretical analysis but also to exhibit other complex dynamics in Section 5. In Section 6, chaos is controlled to an unstable fixed point using the feedback control method. Finally, we conclude this paper with comments and discuss the future work.

\section{The Existence and Stability of Fixed Points}

In this section, we discuss the existence and stability of the fixed points. It is clear that the fixed points of system (4) satisfy the following equations:

$$
\begin{aligned}
& x=x+h[(x+\theta y)(1-x-y)-\delta x-s x y], \\
& y=y+h[s x y-(\delta+\gamma) y] .
\end{aligned}
$$

By calculation of the above system, we get the following results: system (4) has three fixed points $(0,0),(1-\delta, 0)$ and the positive fixed point $\left(x^{*}, y^{*}\right)$, where $\left(x^{*}, y^{*}\right)$ satisfy

$$
\begin{aligned}
\left(x^{*}+\theta y^{*}\right)\left(1-x^{*}-y^{*}\right)-\delta x^{*}-s x^{*} y^{*} & =0, \\
x^{*} & =\frac{\delta+\gamma}{s} .
\end{aligned}
$$

Now we study the stability of these fixed points. Note that the modules of eigenvalues of the characteristic equation at the fixed point determine the local stability of a fixed point $(x, y)$.

The Jacobian matrix $J$ of system (4) at any point $(x, y)$ is given by

$$
J(x, y)=\left(\begin{array}{cc}
1+h[1-2 x-(\theta+1+s) y-\delta] & h[\theta(1-x-y)-(x+\theta y)-s x] \\
h s y & 1+h(s x-\delta-\gamma)
\end{array}\right) .
$$


Let

$$
\begin{aligned}
& a_{11}=1+h(1-x-y-x-\theta y-\delta-s y) ; \\
& a_{12}=h[\theta(1-x-y)-(x+\theta y)-s x] ; \\
& a_{21}=h s y ; \\
& a_{22}=1+h(s x-\delta-\gamma),
\end{aligned}
$$

and then

$$
J(x, y)=\left(\begin{array}{ll}
a_{11} & a_{12} \\
a_{21} & a_{22}
\end{array}\right) .
$$

The characteristic equation of $J(x, y)$ is

$$
\lambda^{2}+p(x, y) \lambda+q(x, y)=0,
$$

where

$$
\begin{aligned}
& p(x, y)=-\left(a_{11}+a_{22}\right), \\
& q(x, y)=a_{11} a_{22}-a_{12} a_{21} .
\end{aligned}
$$

Then we will discuss the local dynamics of three fixed points $(0,0),(1-\delta, 0)$ and the positive fixed point $\left(x^{*}, y^{*}\right)$. In order to discuss the stability of the fixed points of system (4), we get the following existence proposition of the fixed points by simple analysis [17-20].

Case 1. For $(0,0)$, we have

$$
J(0,0)=\left(\begin{array}{cc}
1+h(1-\delta) & h \theta \\
0 & 1-h(\delta+\gamma)
\end{array}\right) .
$$

Since two eigenvalues of $J(0,0)$ are $\lambda_{1}=1+h(1-\delta)>1$ and $\lambda_{2}=1-h(\delta+\gamma)<1$, then $(0,0)$ is a saddle.

Case 2. A simple calculation shows the local dynamics of the fixed point $(1-\delta, 0)$.

Proposition 1. The eigenvalues of the fixed point $(1-\delta, 0)$ are $\lambda_{1}=1+h(\delta-1), \lambda_{2}=1+h[s(1-\delta)-\delta-\gamma]$ :

(i) $(1-\delta, 0)$ is a source if $0<\delta<1, \delta+\gamma\rangle s(1-\delta)$ and $h>\max \{2 /(1-\delta), 2 /(\delta+\gamma-s(1-\delta))\}$;

(ii) $(1-\delta, 0)$ is a sink if $0<\delta<1, \delta+\gamma\rangle s(1-\delta)$ and $0<h<\min \{2 /(1-\delta), 2 /(\delta+\gamma-s(1-\delta))\}$;

(iii) $(1-\delta, 0)$ is not hyperbolic if $0<\delta<1, \delta+\gamma>s(1-\delta)$ and $h=2 /(1-\delta)$ or $h=2 /(\delta+\gamma-s(1-\delta))$;

(iv) $(1-\delta, 0)$ is a saddle except for any values of parameters as (i) to (iii).

Case 3. The characteristic equation of the Jacobian matrix $J$ of system (4) at the positive fixed point $\left(x^{*}, y^{*}\right)$ is written as

$$
\lambda^{2}+\operatorname{tr} J\left(x^{*}, y^{*}\right) \lambda+\operatorname{det} J\left(x^{*}, y^{*}\right)=0,
$$

where

$$
\begin{aligned}
& \operatorname{tr} J\left(x^{*}, y^{*}\right)=-2-h M, \\
& \operatorname{det} J\left(x^{*}, y^{*}\right)=1+h M+h^{2} N, \\
& M=1-2 x^{*}-(\theta+1+s) y^{*}-\delta, \\
& N=s y^{*}\left[\left(x^{*}+\theta y^{*}\right)+s x^{*}-\theta\left(1-x^{*}-y^{*}\right)\right] .
\end{aligned}
$$

Let

$$
F(\lambda)=\lambda^{2}-(2+h M) \lambda+\left(1+h M+h^{2} N\right),
$$

and then

$$
\begin{gathered}
F(1)=h^{2} N>0, \\
F(-1)=4+2 h M+h^{2} N .
\end{gathered}
$$

Proposition 2. Let $\left(x^{*}, y^{*}\right)$ be the positive fixed point of (4):

(i) it is a sink if one of the following conditions holds:

(i.1) $-2 \sqrt{N} \leqslant M<0$ and $0<h<-M / N$;

(i.2) $M<-2 \sqrt{N}$ and $0<h<\left(-M-\sqrt{M^{2}-4 N}\right) / N$;

(ii) it is a source if one of the following conditions holds:

$$
\begin{aligned}
& \text { (ii.1) }-2 \sqrt{N} \leqslant M<0 \text { and } h>-M / N \text {; } \\
& \text { (ii.2) } M<-2 \sqrt{N} \text { and } h>\left(-M+\sqrt{M^{2}-4 N}\right) / N \text {; } \\
& \text { (ii.3) } M \geqslant 0 \text {; }
\end{aligned}
$$

(iii) it is a saddle if the following condition holds:

$$
\begin{gathered}
M<-2 \sqrt{N}, \\
\frac{-M-\sqrt{M^{2}-4 N}}{N}<h<\frac{-M+\sqrt{M^{2}-4 N}}{N} ;
\end{gathered}
$$

(iv) it is nonhyperbolic if one of the following conditions holds:

$$
\begin{aligned}
& \text { (iv.1) } M<-2 \sqrt{N} \text { and } h=\left(-M \pm \sqrt{M^{2}-4 N}\right) / N \text { and } \\
& \delta \neq-2 / M,-4 / M ; \\
& \text { (iv.2) }-2 \sqrt{N} \leqslant M<0 \text { and } h=-M / N \text {. }
\end{aligned}
$$

From the above discussion, we can easily obtain that if (iv.1) of Proposition 2 holds, then one of the eigenvalues of the positive fixed point $\left(x^{*}, y^{*}\right)$ is -1 and the other is neither 1 nor -1 .

Let

$$
\begin{aligned}
F_{1} & =\{(\theta, \delta, s, \gamma, h): M<-2 \sqrt{N}, h \\
& \left.=\frac{-M-\sqrt{M^{2}-4 N}}{N}, \theta, \delta, s, \gamma, h>0\right\}
\end{aligned}
$$


and

$$
\begin{aligned}
F_{2} & =\{(\theta, \delta, s, \gamma, h): M<-2 \sqrt{N}, h \\
& \left.=\frac{-M+\sqrt{M^{2}-4 N}}{N}, \theta, \delta, s, \gamma, h>0\right\} .
\end{aligned}
$$

When parameters vary in a small neighborhood of $F_{1}$ or $F_{2}$, there may be flip bifurcation of the fixed point $\left(x^{*}, y^{*}\right)$.

Then we can get that the eigenvalues of the positive fixed point $\left(x^{*}, y^{*}\right)$ are a pair of conjugate complex numbers with modulus 1 if (iv.2) of Proposition 2 holds.

Let

$$
\begin{aligned}
F_{3} & =\{(\theta, \delta, s, \gamma, h):-2 \sqrt{N}<M<0, h \\
& \left.=\frac{-M}{N}, \theta, \delta, s, \gamma, h>0\right\} .
\end{aligned}
$$

The fixed point $\left(x^{*}, y^{*}\right)$ may undergo Neimark-Sacker bifurcation when parameters vary in a small neighborhood of $F_{3}$.

\section{Bifurcation Analysis}

Based on the above analysis, we will mainly focus on the flip bifurcation of the positive fixed point $\left(x^{*}, y^{*}\right)$ if parameters vary in a small neighborhood of $F_{1}$ or $F_{2}$ and the NeimarkSacker bifurcation of $\left(x^{*}, y^{*}\right)$ if parameters vary in a small neighborhood of $F_{3}$, respectively, to investigate the local and global stability of system (4). We choose parameter $h$ as a bifurcation parameter for studying the flip bifurcation and
Neimark-Sacker bifurcation of $\left(x^{*}, y^{*}\right)$ by using the center manifold theorem and bifurcation theory in [17-20, 33-36].

3.1. Flip Bifurcation. Taking parameters $\left(\theta, \delta, s, \gamma, h_{1}\right)$ arbitrarily from $F_{1}$, we consider system (4) with $\left(\theta, \delta, s, \gamma, h_{1}\right)$, which is described by

$$
\begin{aligned}
& \left(\begin{array}{l}
x \\
y
\end{array}\right) \\
& \longrightarrow\left(\begin{array}{c}
x+h_{1}[(x+\theta y)(1-x-y)-\delta x-s x y] \\
y+h_{1}[s x y-(\delta+\gamma) y]
\end{array}\right) .
\end{aligned}
$$

The map (23) has a unique positive fixed point $\left(x^{*}, y^{*}\right)$, whose eigenvalues are $\lambda_{1}=-1, \lambda_{2}=3+M h_{1}$ with $\left|\lambda_{2}\right| \neq 1$ by Proposition 2 .

Since $\left(\theta, \delta, s, \gamma, h_{1}\right) \in F_{1}, h_{1}=\left(-M-\sqrt{M^{2}-4 N}\right) / N$. Choose $h^{*}$ as a bifurcation parameter, we consider a perturbation of (23) as follows:

$$
\begin{aligned}
& \left(\begin{array}{l}
x \\
y
\end{array}\right) \\
& \longrightarrow\left(\begin{array}{c}
x+\left(h_{1}+h^{*}\right)[(x+\theta y)(1-x-y)-\delta x-s x y] \\
y+\left(h_{1}+h^{*}\right)[s x y-(\delta+\gamma) y]
\end{array}\right),
\end{aligned}
$$

where $\left|h^{*}\right| \ll 1$, which is a small perturbation parameter.

Let $X=x-x^{*}, Y=y-y^{*}$. Then we transform the fixed point $\left(x^{*}, y^{*}\right)$ of map (24) into the origin. By calculating we have

$$
\begin{aligned}
& \left(\begin{array}{l}
X \\
Y
\end{array}\right) \\
& \longrightarrow\left(\begin{array}{c}
a_{11} X+a_{12} Y+a_{13} X^{2}+a_{14} X Y+a_{15} Y^{2}+b_{1} X h^{*}+b_{2} Y h^{*}+b_{3} X^{2} h^{*}+b_{4} X Y h^{*}+b_{5} Y^{2} h^{*}+O\left(\left(|X|+|Y|+\left|h^{*}\right|\right)^{3}\right) \\
a_{21} X+a_{22} Y+a_{23} X Y+c_{1} X h^{*}+c_{2} Y h^{*}+c_{3} X Y h^{*}+O\left(\left(|X|+|Y|+\left|h^{*}\right|\right)^{3}\right)
\end{array}\right),
\end{aligned}
$$

where

$$
\begin{aligned}
& a_{11}=1+h\left(1-2 x^{*}-(\theta+1) y^{*}-\delta-s y^{*}\right), \\
& a_{12}=h\left[\theta\left(1-x^{*}-y^{*}\right)-x^{*}-\theta y^{*}-s x^{*}\right], \\
& a_{13}=-h, \\
& a_{14}=-h(1+\theta+s), \\
& a_{15}=-\theta h, \\
& b_{1}=\left[1-2 x^{*}-(\theta+1) y^{*}-\delta-s y^{*}\right], \\
& b_{2}=\left[\theta\left(1-x^{*}-y^{*}\right)-x^{*}-\theta y^{*}-s x^{*}\right], \\
& b_{3}=-1,
\end{aligned}
$$

$$
\begin{aligned}
b_{4} & =-(1+\theta+s), \\
b_{5} & =-\theta, \\
a_{21} & =h s y^{*}, \\
a_{22} & =1+h s x^{*}, \\
a_{2} 3 & =h s, \\
c_{1} & =s y^{*}, \\
c_{2} & =s x^{*}, \\
c_{3} & =s,
\end{aligned}
$$

and $h=h_{1}$. 
We construct an invertible matrix $T$ as follows:

$$
T=\left(\begin{array}{cc}
a_{12} & a_{12} \\
-1-a_{11} & \lambda_{2}-a_{11}
\end{array}\right),
$$

and for (25), use the translation

$$
\left(\begin{array}{l}
X \\
Y
\end{array}\right)=T\left(\begin{array}{l}
\tilde{x} \\
\tilde{y}
\end{array}\right)
$$

and then the map (25) becomes

$$
\left(\begin{array}{c}
\tilde{x} \\
\tilde{y}
\end{array}\right) \rightarrow\left(\begin{array}{cc}
-1 & 0 \\
0 & \lambda_{2}
\end{array}\right)\left(\begin{array}{l}
\tilde{x} \\
\tilde{y}
\end{array}\right)+\left(\begin{array}{l}
f\left(X, Y, h^{*}\right) \\
g\left(X, Y, h^{*}\right)
\end{array}\right),
$$

where

$$
\begin{aligned}
f\left(X, Y, h^{*}\right)= & \frac{a_{13}\left(\lambda_{2}-a_{11}\right)}{a_{12}\left(\lambda_{2}+1\right)} X^{2} \\
& +\frac{a_{14}\left(\lambda_{2}-a_{11}\right)+a_{12} a_{23}}{a_{12}\left(\lambda_{2}+1\right)} X Y \\
& +\frac{a_{15}\left(\lambda_{2}-a_{11}\right)}{a_{12}\left(\lambda_{2}+1\right)} Y^{2} \\
& +\frac{b_{1}\left(\lambda_{2}-a_{11}\right)-a_{12} c_{1}}{a_{12}\left(\lambda_{2}+1\right)} h^{*} X \\
& +\frac{b_{2}\left(\lambda_{2}-a_{11}\right)-a_{12} c_{2}}{a_{12}\left(\lambda_{2}+1\right)} h^{*} Y \\
& -\frac{\lambda_{2}-a_{11}}{a_{12}\left(\lambda_{2}+1\right)} h^{*} X^{2} \\
& +\frac{b_{4}\left(\lambda_{2}-a_{11}\right)-a_{12} c_{3}}{a_{12}\left(\lambda_{2}+1\right)} h^{*} X Y \\
& +\frac{b_{5}\left(\lambda_{2}-a_{11}\right)}{a_{12}\left(\lambda_{2}+1\right)} h^{*} Y^{2} \\
& +O\left(\left(X|+| Y|+| h^{*} \mid\right)^{3}\right),
\end{aligned}
$$

$$
\begin{aligned}
g\left(X, Y, h^{*}\right)= & \frac{a_{13}\left(a_{11}+1\right)}{a_{12}\left(\lambda_{2}+1\right)} X^{2} \\
& +\frac{a_{14}\left(a_{11}+1\right)+a_{12} a_{23}}{a_{12}\left(\lambda_{2}+1\right)} X Y \\
& +\frac{a_{15}\left(a_{11}+1\right)}{a_{12}\left(\lambda_{2}+1\right)} Y^{2} \\
& +\frac{b_{1}\left(a_{11}+1\right)+a_{12} c_{1}}{a_{12}\left(\lambda_{2}+1\right)} h^{*} X \\
& +\frac{b_{2}\left(a_{11}+1\right)+a_{12} c_{2}}{a_{12}\left(\lambda_{2}+1\right)} h^{*} Y \\
& -\frac{a_{11}+1}{a_{12}\left(\lambda_{2}+1\right)} h^{*} X^{2} \\
& +\frac{b_{4}\left(a_{11}+1\right)+a_{12} c_{3}}{a_{12}\left(\lambda_{2}+1\right)} h^{*} X Y \\
& +\frac{b_{5}\left(a_{11}+1\right)\left(\lambda_{2}-a_{11}\right)}{\left.a_{12}(\lambda)_{2}+1\right)} h^{*} Y^{2} \\
& \left.O\left(|X|+|Y|+\left|h^{*}\right|\right)^{3}\right) \\
& \left.+\frac{1}{1}\right)
\end{aligned}
$$

and

$$
\begin{aligned}
& X=a_{12} \tilde{x}+a_{12} \tilde{y}, \\
& Y=-\left(1+a_{11}\right) \tilde{x}+\left(\lambda_{2}-a_{11}\right) \tilde{y} .
\end{aligned}
$$

From the center manifold theorem [33], we can determine the center manifold $W^{c}(0,0,0)$ of $(29)$ at the fixed point $(0,0)$ in a small neighborhood of $h^{*}=0$, and then we obtain that there exists a center manifold $W^{c}(0,0,0)$, which can be approximately represented as follows:

$$
\begin{aligned}
W^{c}(0,0,0)= & \left(\tilde{x}, \tilde{y}, h^{*}\right) \in R^{3}: \\
\tilde{y}= & a_{1} \tilde{x}^{2}+a_{2} \tilde{x} h^{*}+a_{3} h^{* 2} \\
& +O\left(\left(|\tilde{x}|+\left|h^{*}\right|\right)^{3}\right),
\end{aligned}
$$

where $O\left(\left(|\widetilde{x}|+\left|h^{*}\right|\right)^{3}\right)$ is a function with order at least 3 in the variables, and

$$
\begin{aligned}
& a_{1}=\frac{-a_{12} a_{13}\left(a_{11}+1\right)-a_{12}\left(a_{11}+1\right)^{2}\left[a_{14}\left(a_{11}+1\right)-a_{12} a_{23}\right]-a_{15}\left(a_{11}+1\right)^{3}}{a_{12}\left(1-\lambda_{2}^{2}\right)}, \\
& a_{2}=\frac{\left(a_{11}+1\right)^{2} b_{2}+\left(a_{11}+1\right) a_{12} c_{2}-\left(a_{11}+1\right) a_{12} b_{1}-a_{12}^{2} c_{1}}{\left(\lambda_{2}+1\right)^{2} a_{12}} \\
& a_{3}=0 .
\end{aligned}
$$


Therefore, the map (29) which is restricted to the center manifold $W^{c}(0,0,0)$ is defined:

$$
\begin{aligned}
F: \tilde{x} \longrightarrow & -\tilde{x}+k_{1} \tilde{x}^{2}+k_{2} \tilde{x} h^{*}+k_{3} \tilde{x}^{2} h^{*}+k_{4} \tilde{x} h^{* 2} \\
& +k_{5} \tilde{x}^{3}+O\left(\left(|\tilde{x}|+\left|h^{*}\right|\right)^{4}\right),
\end{aligned}
$$

where

$$
\begin{aligned}
& k_{1}=\frac{\left(\lambda_{2}-a_{11}\right) a_{13}}{\lambda_{2}+1} \\
& +\frac{\left(a_{11}+1\right)\left[a_{12} a_{23}-a_{14}\left(\lambda_{2}-a_{11}\right)\right]}{\lambda_{2}+1} \\
& +\frac{\left(a_{11}+1\right)^{2}\left(\lambda_{2}-a_{11}\right) a_{15}}{\left(\lambda_{2}+1\right) a_{12}} \\
& k_{2}=\frac{\left(\lambda_{2}-a_{11}\right) b_{1}-a_{12} c_{1}}{\lambda_{2}+1} \\
& -\frac{\left(a_{11}+1\right)\left[b_{2}\left(\lambda_{2}-a_{11}\right)-a_{12} c_{2}\right]}{\left(\lambda_{2}+1\right) a_{12}}, \\
& k_{3}=\frac{\left(\lambda_{2}-a_{11}\right) b_{1}-a_{12} c_{1}}{\lambda_{2}+1} a_{1} \\
& +\frac{b_{2}\left(\lambda_{2}-a_{11}\right)-a_{12} c_{2}}{\left(\lambda_{2}+1\right) a_{12}}\left(\lambda_{2}-a_{11}\right) a_{1} \\
& +\frac{\left(1+a_{11}\right)^{2}\left(\lambda_{2}-a_{11}\right) b_{5}}{a_{12}\left(\lambda_{2}+1\right)}+\frac{2 a_{13} a_{12} a_{1}}{\lambda_{2}+1} \\
& -\frac{4 a_{15} a_{2}\left(1+a_{11}\left(\lambda_{2}-a_{11}\right)^{2}\right)}{a_{12}\left(\lambda_{2}+1\right)} \\
& +\frac{\left(\lambda_{2}-a_{11}\right) a_{14}-a_{12} a_{23}}{a_{12}\left(\lambda_{2}+1\right)}\left[a_{12}\left(\lambda_{2}-a_{11}\right)\right. \\
& \left.-a_{11}\left(1+a_{11}\right)\right] 2 a_{2} \text {, } \\
& k_{4}=\frac{\left(\lambda_{2}-a_{11}\right) b_{1}-a_{12} c_{1}}{\lambda_{2}+1} a_{1} \\
& +\frac{b_{2}\left(\lambda_{2}-a_{11}\right)-a_{12} c_{2}}{\left(\lambda_{2}+1\right) a_{12}}\left(\lambda_{2}-a_{11}\right) a_{2}, \\
& k_{5}=\frac{2 a_{13} a_{12} a_{1}}{\lambda_{2}+1} \\
& +\frac{\left(\lambda_{2}-a_{11}\right) a_{14}-a_{12} a_{23}}{a_{12}\left(\lambda_{2}+1\right)}\left[a_{12}\left(\lambda_{2}-a_{11}\right)\right. \\
& \left.-a_{11}\left(1+a_{11}\right)\right] a_{1}-\frac{2 a_{15} a_{1}\left(1+a_{11}\left(\lambda_{2}-a_{11}\right)^{2}\right)}{a_{12}\left(\lambda_{2}+1\right)} .
\end{aligned}
$$

For map (34) in order to undergo a flip bifurcation, we require that two discriminatory quantities $\alpha_{1}$ and $\alpha_{2}$ are not zero, where

$$
\alpha_{1}=\left.\left(\frac{\partial^{2} F}{\partial \tilde{x} \partial h^{*}}+\frac{1}{2} \frac{\partial F}{\partial h^{*}} \frac{\partial^{2} F}{\partial \tilde{x}^{2}}\right)\right|_{(0,0)}=k_{2}
$$

and

$$
\alpha_{2}=\left.\left(\frac{1}{6} \frac{\partial^{3} F}{\partial \tilde{x}^{3}}+\left(\frac{1}{2} \frac{\partial^{2} F}{\partial \tilde{x}^{2}}\right)^{2}\right)\right|_{(0,0)}=k_{5}+k_{1}^{2} .
$$

On the basis of the above analysis and the theorem of [3436], we obtain the following result.

Theorem 3. If $\alpha_{2} \neq 0$, then map (4) undergoes a flip bifurcation at the fixed point $\left(x^{*}, y^{*}\right)$ when the parameter $h$ varies in a small neighborhood of $h_{1}$. Moreover, if $\alpha_{2}>0$ (resp., $\left.\alpha_{2}<0\right)$, the period -2 orbits that bifurcate from $\left(x^{*}, y^{*}\right)$ are stable (resp., unstable).

3.2. Neimark-Sacker Bifurcation. In this section, we can give a similar argument with the flip bifurcation. Taking parameters $\left(\theta, \delta, s, \gamma, h_{2}\right)$ arbitrarily from $F_{3}$, we consider system (4) with $\left(\theta, \delta, s, \gamma, h_{2}\right)$, which is described by

$$
\begin{aligned}
& \left(\begin{array}{l}
x \\
y
\end{array}\right) \\
& \longrightarrow\left(\begin{array}{c}
x+h_{2}[(x+\theta y)(1-x-y)-\delta x-s x y] \\
y+h_{2}[s x y-(\delta+\gamma) y]
\end{array}\right) .
\end{aligned}
$$

The map (38) has a unique positive fixed point $\left(x^{*}, y^{*}\right)$.

Since $\left(\theta, \delta, s, \gamma, h_{2}\right) \in F_{3}, h_{2}=-M / N$. We give a perturbation $\bar{h}^{*}$ at $h_{2}$. Then (38) becomes into the following form:

$$
\begin{aligned}
& \left(\begin{array}{l}
x \\
y
\end{array}\right) \\
& \longrightarrow\left(\begin{array}{c}
x+\left(h_{2}+\bar{h}^{*}\right)[(x+\theta y)(1-x-y)-\delta x-s x y] \\
y+\left(h_{2}+\bar{h}^{*}\right)[s x y-(\delta+\gamma) y]
\end{array}\right),
\end{aligned}
$$

where $\left|\bar{h}^{*}\right| \ll 1$, which is a small perturbation parameter.

Let $X=x-x^{*}, Y=y-y^{*}$. Then we transform the fixed point $\left(x^{*}, y^{*}\right)$ of map (39) into the origin, and we have 


$$
\left(\begin{array}{l}
X \\
Y
\end{array}\right) \longrightarrow\left(\begin{array}{c}
a_{11} X+a_{12} Y+a_{13} X^{2}+a_{14} X Y+a_{15} Y^{2}+O\left(\left(|X|+|Y|+\left|h^{*}\right|\right)^{3}\right) \\
a_{21} X+a_{22} Y+a_{23} X Y+O\left(\left(|X|+|Y|+\left|h^{*}\right|\right)^{3}\right)
\end{array}\right)
$$

where $a_{11}, a_{12}, a_{13}, a_{14}, a_{15}, a_{21}, a_{22}, a_{23}$ are given in (26) by substituting $h$ for $h_{2}+\bar{h}^{*}$.

Note that the characteristic equation associated with the linearization of the map $(40)$ at $(X, Y)=(0,0)$ is following

$$
\lambda^{2}+p\left(\bar{h}^{*}\right) \lambda+q\left(\bar{h}^{*}\right)=0,
$$

where

$$
\begin{aligned}
& p\left(\bar{h}^{*}\right)=-2-\left(h+\bar{h}^{*}\right) M, \\
& q\left(\bar{h}^{*}\right)=1+\left(h+\bar{h}^{*}\right) M+\left(h+\bar{h}^{*}\right)^{2} N .
\end{aligned}
$$

Since parameters $\left(\theta, \delta, s, \gamma, h_{2}\right) \in F_{3}$, the eigenvalues of $(0,0)$ are a pair of complex conjugate numbers $\lambda$ and $\bar{\lambda}$ with modulus 1 by Proposition 2, where

$$
\begin{aligned}
\lambda, \bar{\lambda} & =-\frac{p\left(\bar{h}^{*}\right)}{2} \pm \frac{i}{2} \sqrt{4 q\left(\bar{h}^{*}\right)-p^{2}\left(\bar{h}^{*}\right)} \\
& =1+\frac{M\left(h_{2}+\bar{h}^{*}\right)}{2} \pm \frac{i\left(h_{2}+\bar{h}^{*}\right)}{2} \sqrt{4 N-M^{2}}
\end{aligned}
$$

and we have

$$
\begin{array}{r}
|\lambda|=\sqrt{q\left(\bar{h}^{*}\right)}, \\
l=\left.\frac{\mathrm{d}|\lambda|}{\mathrm{d} \bar{h}^{*}}\right|_{\bar{h}^{*}=0}=-\frac{M}{2}>0 .
\end{array}
$$

Moreover, it is required that when $\bar{h}^{*}=0, \lambda^{m}, \bar{\lambda}^{m} \neq 1$ $(m \neq 1,2,3,4)$ which is equivalent to $p(0) \neq-2,0,1,2$. Note that $\left(\theta, \delta, s, \gamma, h_{2}\right) \in F_{3}$. Thus, $p(0) \neq-2,2$. We only need to require that $p(0) \neq 0,1$, which leads to

$$
M^{2} \neq 2 N, 3 N \text {. }
$$

Hence, the eigenvalues $\lambda, \bar{\lambda}$ of fixed point $(0,0)$ of $(40)$ do not lie in the intersection of the unit circle with the coordinate axes when $\bar{h}^{*}=0$ and (45) holds.

Next, we discuss the normal form of $(40)$ at $\bar{h}^{*}=0$.

Let $\bar{h}^{*}=0, \mu=1+M h_{2} / 2, \omega=\left(h_{2} / 2\right) \sqrt{4 N-M^{2}}$,

$$
T=\left(\begin{array}{cc}
a_{12} & 0 \\
\mu-a_{11} & -\omega
\end{array}\right),
$$

and then $T$ is invertible and use the translation

$$
\left(\begin{array}{l}
X \\
Y
\end{array}\right)=T\left(\begin{array}{l}
\tilde{x} \\
\tilde{y}
\end{array}\right)
$$

for (40); then model (40) becomes the following form:

$$
\left(\begin{array}{l}
\tilde{x} \\
\tilde{y}
\end{array}\right) \longrightarrow\left(\begin{array}{cc}
\mu & -\omega \\
\omega & \mu
\end{array}\right)\left(\begin{array}{l}
\tilde{x} \\
\tilde{y}
\end{array}\right)+\left(\begin{array}{l}
\tilde{f}(\tilde{x}, \tilde{y}) \\
\tilde{g}(\tilde{x}, \tilde{y})
\end{array}\right),
$$

where

$$
\begin{aligned}
\tilde{f}(\tilde{x}, \tilde{y})= & \frac{a_{13}}{a_{12}} X^{2}+\frac{a_{14}}{a_{12}} X Y+\frac{a_{15}}{a_{12}} Y^{2} \\
& +O\left(\left(|X|+|Y|+\left|h^{*}\right|\right)^{3}\right), \\
\tilde{g}(\tilde{x}, \tilde{y})= & \frac{a_{13}\left(\mu-a_{11}\right)}{a_{12} \omega} X^{2} \\
& +\left(\frac{a_{14}\left(\mu-a_{11}\right)}{a_{12} \omega}-\frac{a_{23}}{\omega}\right) X Y \\
& +\frac{a_{15}\left(\mu-a_{11}\right)}{a_{12} \omega} Y^{2} \\
& +O\left(\left(|X|+|Y|+\left|h^{*}\right|\right)^{3}\right), \\
X= & a_{12} \tilde{x}, \\
Y= & \left(\mu-a_{11}\right) \tilde{x}-\omega \tilde{y} .
\end{aligned}
$$

In order to undergo Neimark-Sacker bifurcation for (48), we require that the following discriminatory quantity is not zero [34-36]:

$$
\begin{aligned}
b= & {\left[-\operatorname{Re}\left(\frac{(1-2 \lambda) \bar{\lambda}^{2}}{1-\lambda} \xi_{20} \xi_{11}\right)-\frac{1}{2}\left|\xi_{11}\right|^{2}-\left|\xi_{02}\right|^{2}\right.} \\
& \left.+\operatorname{Re}\left(\bar{\lambda} \xi_{21}\right)\right]\left.\right|_{\bar{h}^{*}=0},
\end{aligned}
$$

where

$$
\begin{aligned}
\xi_{20} & =\frac{1}{8}\left[\left(\tilde{f}_{\tilde{x} \tilde{x}}-\tilde{f}_{\tilde{y} \tilde{y}}+2 \widetilde{g}_{\tilde{x} \tilde{y}}\right)+i\left(\tilde{g}_{\tilde{x} \tilde{x}}-\tilde{g}_{\tilde{y} \tilde{y}}-2 \tilde{f}_{\tilde{x} \tilde{y}}\right)\right], \\
\xi_{11} & =\frac{1}{4}\left[\left(\widetilde{f}_{\tilde{x} \tilde{x}}+\widetilde{f}_{\tilde{y} \tilde{y}}\right)+i\left(\widetilde{g}_{\tilde{x} \tilde{x}}+\widetilde{g}_{\tilde{y} \tilde{y}}\right)\right], \\
\xi_{02} & =\frac{1}{8}\left[\left(\tilde{f}_{\tilde{x} \tilde{x}}-\tilde{f}_{\tilde{y} \tilde{y}}-2 \widetilde{g}_{\tilde{x} \tilde{y}}\right)+i\left(\tilde{g}_{\tilde{x} \tilde{x}}-\widetilde{g}_{\tilde{y} \tilde{y}}+2 \tilde{f}_{\tilde{x} \tilde{y}}\right)\right], \\
\xi_{21} & =\frac{1}{16}\left[\left(\tilde{f}_{\tilde{x} \tilde{x} \tilde{x}}+\tilde{f}_{\tilde{x} \tilde{y} \tilde{y}}+\tilde{g}_{\tilde{x} \tilde{x} \tilde{y}}+\tilde{g}_{\tilde{y} \tilde{y} \tilde{y}}\right)\right. \\
& \left.+i\left(\tilde{g}_{\tilde{x} \tilde{x} \tilde{x}}+\tilde{g}_{\tilde{x} \tilde{y} \tilde{y}}-\tilde{f}_{\tilde{x} \tilde{x} \tilde{y}}-\tilde{f}_{\tilde{y} \tilde{y} \tilde{y}}\right)\right] .
\end{aligned}
$$


Thus, some complicated calculation gives

$$
\begin{aligned}
& b=-\frac{1}{32\left((1-\mu)^{2}+\omega^{2}\right)}\left[m \left(\widetilde{f}_{\tilde{x} \tilde{x}}^{2}-\tilde{g}_{\tilde{x} \tilde{x}}^{2}+\tilde{g}_{\tilde{y} \tilde{y}}^{2}\right.\right. \\
& \left.+2 \tilde{f}_{\tilde{x} \tilde{x}} \tilde{g}_{\tilde{x} \tilde{y}}+2 \tilde{f}_{\tilde{x} \tilde{y}} \tilde{g}_{\tilde{x} \tilde{x}}+\tilde{f}_{\tilde{x} \tilde{y}} \tilde{g}_{\tilde{y} \tilde{y}}\right)+n\left(2 \tilde{f}_{\tilde{x} \tilde{x}} \tilde{g}_{\tilde{x} \tilde{x}}\right. \\
& \left.\left.-2 \widetilde{f}_{\tilde{x} \tilde{x}} \tilde{f}_{\tilde{x} \tilde{y}}+2 \widetilde{g}_{\tilde{x} \tilde{x}} \tilde{g}_{\tilde{x} \tilde{y}}+2 \widetilde{g}_{\tilde{y} \tilde{y}} \widetilde{g}_{\tilde{x} \tilde{y}}\right)\right]-\frac{1}{64}\left[3 \widetilde{f}_{\tilde{x} \tilde{x}}^{2}\right. \\
& +3 \widetilde{g}_{\tilde{x} \tilde{x}}^{2}+3 \widetilde{g}_{\tilde{y} \tilde{y}}^{2}+4 \widetilde{f}_{\tilde{x} \tilde{y}}^{2}+4 \widetilde{g}_{\tilde{x} \tilde{y}}^{2}-4 \widetilde{f}_{\tilde{x} \tilde{x}} \widetilde{g}_{\tilde{x} \tilde{y}} \\
& -4 \widetilde{f}_{\tilde{x} \tilde{y}} \widetilde{g}_{\tilde{y} \tilde{y}}+4 \tilde{f}_{\tilde{x} \tilde{y}} \tilde{g}_{\tilde{x} \tilde{x}}+2 \widetilde{g}_{\tilde{x} \tilde{x}} \widetilde{g}_{\tilde{y} \tilde{y}}-4 \mu\left(\tilde{f}_{\tilde{x} \tilde{x} \tilde{x}}\right. \\
& \left.\left.+\widetilde{g}_{\tilde{x} \tilde{x} \tilde{y}}\right)-4 \omega\left(\widetilde{g}_{\tilde{x} \tilde{x} \tilde{x}}+\widetilde{g}_{\tilde{x} \tilde{y} \tilde{y}}-\tilde{f}_{\tilde{x} \tilde{x} \tilde{y}}\right)\right],
\end{aligned}
$$

where

$$
\begin{aligned}
& m=\mu^{2}-3 \mu^{3}+2 \mu^{4}-\omega^{2}+\mu \omega^{2}-2 \omega^{4}, \\
& n=-\omega\left(\omega^{2}+5 \mu^{2}-2 \mu-4 \mu^{3}-4 \mu \omega^{2}\right), \\
& \tilde{f}_{\tilde{x} \tilde{x}}=2\left[a_{12} a_{13}+a_{14}\left(\mu-a_{11}\right)+\frac{a_{15}}{a_{12}}\left(\mu-a_{11}\right)^{2}\right] \\
& \tilde{f}_{\tilde{x} \tilde{y}}=a_{14} \omega-\frac{2 a_{15} \omega\left(\mu-a_{11}\right)}{a_{12}}, \\
& \tilde{f}_{\tilde{y} \tilde{y}}=\frac{2 a_{15} \omega^{2}}{a_{12}}, \\
& \tilde{f}_{\tilde{x} \tilde{x} \tilde{x}}=\tilde{f}_{\tilde{x} \tilde{x} \tilde{y}}=\tilde{f}_{\tilde{x} \tilde{y} \tilde{y}}=\tilde{f}_{\tilde{y} \tilde{y} \tilde{y}}=0, \\
& \tilde{g}_{\tilde{x} \tilde{x}}=2\left[\frac{a_{12} a_{13}\left(\mu-a_{11}\right)}{\omega}\right. \\
& \tilde{g}_{\tilde{y} \tilde{y}}=\frac{2 a_{15}\left(\mu-a_{11}\right) \omega}{a_{12}}, \\
& \quad+a_{12}\left(\mu-a_{11}\right)\left(\frac{a_{14}\left(\mu-a_{11}\right)}{a_{12} \omega}-\frac{a_{23}}{\omega}\right) \\
& \quad+\frac{a_{15}\left(\mu-a_{11}\right)^{3}}{\tilde{g}_{\tilde{x} \tilde{y}} \omega}, \\
& \tilde{g}_{\tilde{x} \tilde{x} \tilde{y}}=\tilde{g}_{\tilde{x} \tilde{y} \tilde{y}}=\tilde{g}_{\tilde{y} \tilde{y} \tilde{y}}=0 . \\
& a_{12} \omega \\
& \tilde{a}_{12}
\end{aligned}
$$

Therefore, from the above analysis and the theorem in [34-36], we have the following result.

Theorem 4. If condition (45) holds and $b \neq 0$, then map (4) undergoes Neimark-Sacker bifurcation at the fixed point $\left(x^{*}, y^{*}\right)$ when the parameter $h$ varies in a small neighborhood of $h_{2}$. Moreover, if $b<0$, (resp., $b>0$ ), then an attracting (resp., repelling) invariant closed curve bifurcates from the fixed point for $h>h_{2}$ (resp., $h<h_{2}$ ).

\section{Existence of Marotto's Chaos}

In this section, we rigorously prove map (4) possesses chaotic behavior in the sense of Marotto's definition [32].

We first present Marotto's chaos definitions and theorem which are quoted from [32].

For any map $F: R^{n} \rightarrow R^{n}$, and any positive integer $K$, let $F^{K}$ represent the composition of $F$ with itself $K$ times. For a differentiable function $F$, let $D F(Z)$ denote the Jacobian matrix of $F$ evaluated at the point $Z \in R^{n}$, and $|D F(Z)|$ its determinant. Let $B_{r}(Z)$ denote the closed ball in $R^{n}$ of radius $r$ centered at the point $Z$ and $B_{r}^{0}(Z)$ its interior. Also let $\|Z\|$ be the usual Euclidean norm of $Z$ in $R^{n}$.

Definition 5. Let $F$ be differentiable in $B_{r}\left(Z_{0}\right)$. The point $Z_{0} \in$ $R^{n}$ is an expanding fixed point of $F$ in $B_{r}\left(Z_{0}\right)$, if $F\left(Z_{0}\right)=Z_{0}$ and all eigenvalues of $D F(Z)$ exceed 1 in norm for all $Z \in$ $B_{r}\left(Z_{0}\right)$.

Definition 6. Assume that $Z_{0}$ is an expanding fixed point of $F$ in $B_{r}\left(Z_{0}\right)$ for some $r>0$; then $Z_{0}$ is said to be a snap-back repeller of $F$ if there exists a point $\bar{Z} \in B_{r}\left(Z_{0}\right)$ with $\bar{Z} \neq Z_{0}$, $F^{M}(\bar{Z})=Z_{0}$ and $\left|D F^{M}(\bar{Z})\right| \neq 0$ for some positive integer $M$.

Theorem 7. [32] If F possesses a snap-back repeller, then the map $F$ is chaotic. That is, there exist

(i) a positive integer $N$ such that for each integer $P \geqslant N$, $F$ has a point of period $P$;

(ii) a "scrambled set" of $F$, that is, an uncountable set $S$ containing no periodic points of F such that

(a) $F[S] \subset S$,

(b) for every $X, Y \in S$ with $X \neq Y$

$$
\lim _{k \rightarrow \infty} \sup \left\|F^{k}(X)-F^{k}(Y)\right\|>0
$$

(c) for every $X \in S$ and any periodic point $Y$ of $F$

$$
\lim _{k \rightarrow \infty} \sup \left\|F^{k}(X)-F^{k}(Y)\right\|>0
$$

(iii) an uncountable subset $S_{0}$ of $S$ such that for every $X, Y \in$ $S_{0}$

$$
\lim _{k \rightarrow \infty} \inf \left\|F^{k}(X)-F^{k}(Y)\right\|=0 .
$$

Now we theoretically give the condition of existence of chaotic phenomena for map (4) in the sense of Marotto's definition of chaos.

Suppose $Z_{0}\left(x_{0}, y_{0}\right)$ be the fixed point of map (4). We firstly give the conditions such that the $Z_{0}$ is a snap-back repeller. The eigenvalues associated with the fixed point $Z_{0}$ are given by

$$
\lambda_{1,2}=\frac{-p\left(x_{0}, y_{0}\right) \pm \sqrt{p^{2}\left(x_{0}, y_{0}\right)-4 q\left(x_{0}, y_{0}\right)}}{2}
$$


where

$$
\begin{aligned}
& p(x, y)=-2-h[1-2 x-(\theta+1+s) y-\delta+s x-\delta \\
& \quad-\gamma] \\
& q(x, y)=1+h[1-2 x-(\theta+1+s) y-\delta+s x-\delta \\
& \quad-\gamma] \\
& \quad+h^{2}\{[1-2 x-(\theta+1+s) y-\delta](s x-\delta-\gamma) \\
& \quad-s y[\theta(1-x-y)-(x+\theta y)-s x]\} .
\end{aligned}
$$

According to Definition 5, we begin to find a neighborhood $B_{r}\left(Z_{0}\right)$ of $Z_{0}$ in which the norms of conjugate complex eigenvalues exceed 1 for all $Z \in B_{r}\left(Z_{0}\right)$, which are equivalent to

$$
\begin{array}{r}
p^{2}(x, y)-4 q(x, y)<0, \\
q(x, y)-1>0 .
\end{array}
$$

Let $s_{1}(x, y)=p^{2}(x, y)-4 q(x, y)$.

It is easy to see that $s_{1}(x, y)=h^{2}\left[A_{1} y^{2}+B_{1} y+C_{1}\right]$, where

$$
\begin{aligned}
& A_{1}=(\theta+1+s)^{2}-8 s \theta, \\
& B_{1}=2(\theta+1+s)(2 x-\gamma-1-s x)+4 s \theta, \\
& C_{1}=(1-2 x-\delta-s x-\delta-\gamma)^{2}
\end{aligned}
$$

If $\Delta_{1}=B_{1}^{2}-4 A_{1} C_{1}=(4 s \theta)^{2}+16 s \theta(\theta+1+s)(2 x-\gamma-1-$ $s x)+32 s \theta(1-2 x-s x+\gamma)^{2} \geqslant 0$,

then the equation $s_{1}(x, y)=0$ has one real root with multiplicity 2 or two real roots denoted as $\bar{y}_{1}=\left(-B_{1}-\right.$ $\left.\sqrt{B_{1}^{2}-4 A_{1} C_{1}}\right) / 2 A_{1}$ and $\bar{y}_{2}=\left(-B_{1}+\sqrt{B_{1}^{2}-4 A_{1} C_{1}}\right) / 2 A_{1}$.

And $\Delta_{1} \geqslant 0 ;$ we can get

$$
\begin{aligned}
& (\theta+s+1)^{2}-8 s \theta>0 \\
& s \theta+(2 x-\gamma-1-s x) \\
& \quad \cdot[\theta+1+s+2(2 x-\gamma-1-s x)] \geqslant 0 .
\end{aligned}
$$

And $s_{1}(x, y)<0$ for $x \in K_{1}=\{x \mid s \theta+(2 x-\gamma-1-$ $s x)[\theta+1+s+2(2 x-\gamma-1-s x)] \geqslant 0\}$ and $y \in D_{1}=\left(\bar{y}_{1}, \bar{y}_{2}\right)$.

Let $s_{2}(x, y)=q(x, y)-1=h\left(A_{2} y^{2}+B_{2} y+C_{2}\right)$ where

$A_{2}=2 h s \theta$,

$B_{2}$

$$
\begin{aligned}
= & h\left[s^{2} x+s x-s \theta(1-x)-(\theta+1+s)(s x-\delta-\gamma)\right] \\
& -(\theta+1+s),
\end{aligned}
$$

$\mathrm{C}_{2}$

$$
\begin{aligned}
= & 1-2 x-\delta+s x-\delta-\gamma \\
& +h(1-2 x-\delta)(s x-\delta-\gamma) .
\end{aligned}
$$

Under the conditions

$$
\begin{aligned}
x & \in K_{2}=\left\{x \mid(2+s) \delta+2 \gamma-s>0, x>\frac{\delta+\gamma}{s}, x\right. \\
& <\frac{1-\delta}{2} \text { or }(2+s) \delta+2 \gamma-s<0, x>\frac{1-\delta}{2}, x \\
& \left.<\frac{\delta+\gamma}{s}\right\}
\end{aligned}
$$

and $\Delta_{2}=B_{2}^{2}-4 A_{2} C_{2} \geqslant 0$, the equation $s_{2}(x, y)=0$ has one real root with multiplicity 2 or two real roots denoted as $\bar{y}_{3}=\left(-B_{2}-\sqrt{B_{2}^{2}-4 A_{2} C_{2}}\right) / 2 A_{2}$ and $\bar{y}_{4}=\left(-B_{2}+\right.$ $\left.\sqrt{B_{2}^{2}-4 A_{2} C_{2}}\right) / 2 A_{2}$.

And $s_{2}(x, y)>0$ for all $x \in K_{2}$ and $y \in D_{2}=\left(-\infty, \bar{y}_{3}\right) \cup$ $\left(\bar{y}_{4},+\infty\right)$.

Lemma 8. If the conditions (61) and (63) hold, and the $\bar{y}_{1}<\bar{y}_{3}$ or $\bar{y}_{4}<\bar{y}_{2}$, then $D_{1} \cap D_{2} \neq \varnothing$.

Moreover, if one of above conditions holds and the $y$ coordinate of fixed point $Z_{0}\left(x_{0}, y_{0}\right), y_{0}$, satisfies $y_{0} \in D_{y_{0}}^{*} \subset$ $D_{1} \cap D_{2}$, then $Z_{0}\left(x_{0}, y_{0}\right)$ is expanding fixed point of (4) in $U_{Z_{0}}=\left\{(x, y) \mid x \in K_{1} \cap K_{2}, y \in D_{y_{0}}^{*}\right\}$.

Due to Definition 2 of snap-back repeller, we need to find one point $Z \in B_{r}\left(Z_{0}\right)$ such that $Z \neq Z_{0}, F^{M}(Z)=Z_{0}$, $|D F(Z)| \neq 0$ for some positive integer $M$.

In fact, we have

$$
\begin{aligned}
x_{1} & +h\left[\left(x_{1}+\theta y_{1}\right)\left(1-x_{1}-y_{1}\right)-\delta x_{1}-s x_{1} y_{1}\right] \\
& =x_{2}, \\
y_{1} & +h\left[s x_{1} y_{1}-(\delta+\gamma) y_{1}\right]=y_{2},
\end{aligned}
$$

and

$$
\begin{array}{r}
x_{2}+h\left[\left(x_{2}+\theta y_{2}\right)\left(1-x_{2}-y_{2}\right)-\delta x_{2}-s x_{2} y_{2}\right]=x_{0} \\
y_{2}+h\left[s x_{2} y_{2}-(\delta+\gamma) y_{2}\right]=y_{0} .
\end{array}
$$

Now a $F^{2}$ map has been constructed to map the point $Z_{1}\left(x_{1}, y_{1}\right)$ to the fixed point $Z_{0}\left(x_{0}, y_{0}\right)$ after two iterations if there are solutions different from $Z_{0}$ for (64) and (65). By the calculation, the solutions different from $Z_{0}$ for (65) satisfy the following equation:

$$
\begin{aligned}
& E_{1} x_{2}^{4}+E_{2} x_{2}^{3}+E_{3} x_{2}^{2}+E_{4} x_{2}+E_{5}=0 \\
& y_{2}=\frac{y_{0}}{1+h\left(s x_{1}-\delta-\gamma\right)},
\end{aligned}
$$


where

$$
\begin{aligned}
E_{1} & =h^{3} s^{2}, \\
E_{2} & =2 h^{2} s(1-h \delta-h \gamma)-h^{3} s^{2}, \\
E_{3} & =h(1-h \delta-h \gamma)^{2}+h^{2} s y_{0}-h^{2} s \theta y_{0}+h^{2} s \delta \\
& +h^{2} s^{2} y_{0}-2 h^{2} s(1-h \delta-h \gamma), \\
E_{4} & =h(h \delta+h \gamma \\
& -1\left(1-h \delta-h \gamma-y_{0}-\theta y_{0}-\delta-s y_{0}\right)-h^{2} s \theta y_{0} \\
& -1), \\
E_{5} & =h \theta y_{0}(1-h \delta-h \gamma)-h \theta y_{0}^{2}-x_{0} .
\end{aligned}
$$

Substituting $x_{2}$ and $y_{2}$ into (64) and solving $x_{1}, y_{1}$, one gets

$$
\begin{aligned}
& E_{1}^{\prime} x_{1}^{4}+E_{2}^{\prime} x_{1}^{3}+E_{3}^{\prime} x_{1}^{2}+E_{4}^{\prime} x_{1}+E_{5}^{\prime}=0 \\
& y_{1}=\frac{y_{2}}{1+h\left(s x_{1}-\delta-\gamma\right)}
\end{aligned}
$$

where

$$
\begin{aligned}
E_{1}^{\prime} & =h^{3} s^{2}, \\
E_{2}^{\prime} & =2 h^{2} s(1-h \delta-h \gamma)-h^{3} s^{2}, \\
E_{3}^{\prime} & =h(1-h \delta-h \gamma)^{2}+h^{2} s y_{2}-h^{2} s \theta y_{2}+h^{2} s \delta \\
& +h^{2} s^{2} y_{2}-2 h^{2} s(1-h \delta-h \gamma),
\end{aligned}
$$

$$
\begin{aligned}
E_{4}^{\prime} & =h(h \delta+h \gamma \\
& -1\left(1-h \delta-h \gamma-y_{2}-\theta y_{2}-\delta-s y_{2}\right)-h^{2} s \theta y_{2} \\
& -1) \\
E_{5}^{\prime} & =h \theta y_{2}(1-h \delta-h \gamma)-h \theta y_{2}^{2}-x_{2} .
\end{aligned}
$$

Obviously, if the condition in Lemma 8 is satisfied, the solutions of (64) and (65) satisfies (66) and (68). And the condition of $Z_{1}\left(x_{1}, y_{1}\right), Z_{2}\left(x_{2}, y_{2}\right) \neq Z_{0}\left(x_{0}, y_{0}\right), Z_{1}\left(x_{1}, y_{1}\right) \in$ $U_{Z_{0}}$, and $\left\|D F^{2}\left(Z_{1}\right)\right\| \neq 0$ holds; then $Z_{0}$ is a snap-back repeller in $U_{Z_{0}}$. Thus, the following theorem is established.

Theorem 9. Assume that $Z_{0}\left(x_{0}, y_{0}\right)$ is an expanding fixed point in $U_{Z_{0}}$, if the solutions $\left(x_{1}, y_{1}\right),\left(x_{2}, y_{2}\right)$ of (64) and (65) satisfy $\left(x_{1}, y_{1}\right),\left(x_{2}, y_{2}\right) \neq\left(x_{0}, y_{0}\right),\left(x_{1}, y_{1}\right) \in U_{Z_{0}}$ and $\left\|D F^{2}\left(Z_{1}\right)\right\| \neq 0$. Then $Z_{0}\left(x_{0}, y_{0}\right)$ is a snap-back repeller of map (4), and hence map (4) is chaotic in the sense of Marotto.

Next, we give specific values of the parameters for illustrating the existence of conditions in Theorem 9.

Example 10. For $\theta=0.2, s=2.25, \delta=0.005, \gamma=$ $0.465, h=2.5$, system (4) has one fixed point $Z_{0}\left(x_{0}, y_{0}\right)=$ $(0.376,0.2981)$. Based on Lemma 8 and Theorem 9 , we find that region of $Z_{0}$ is $U_{Z_{0}}=\left\{(x, y) \mid 0.4975<x<2, \bar{y}_{1}<\right.$ $\left.y<\bar{y}_{3}\right\} \varsubsetneqq\left\{(x, y) \mid x \in K_{1} \cap K_{2}, y \in D_{1} \cap D_{2}\right\}$, and a point $Z_{1}\left(x_{1}, y_{1}\right)=(1.19535,0.0309312)$ such that $F^{2}\left(Z_{1}\right)=Z_{0}$ and $\left\|D F^{2}\left(Z_{1}\right)\right\| \neq 0$, where

$$
\begin{aligned}
& \bar{y}_{1}=\frac{1.725 x+8.3085-\sqrt{(1.725 x+8.3085)^{2}-33.21(1.465-4.25 x)^{2}}}{16.605} \\
& \bar{y}_{3}=\frac{5.2125-\sqrt{21.725 x-6.7635}}{4.5} .
\end{aligned}
$$

By simple calculation, we can get $Z_{0}\left(x_{0}, y_{0}\right), Z_{1}\left(x_{1}, y_{1}\right) \in$ $U_{Z_{0}}$. Thus, $Z_{0}\left(x_{0}, y_{0}\right)$ is a snap-back repeller.

\section{Numerical Simulations}

In the section, we use Maximum Lyapunov exponents, the bifurcation diagrams, and phase portraits for system (4) to confirm the above theoretical analysis and show the new interesting complex dynamical behaviors. The bifurcation parameters are considered in the following three cases:

(i) varying $h$ in the range $2.7 \leqslant h \leqslant 3.5$, and fixing $\theta=$ $0.6, s=0.2, \delta=0.0011, \gamma=0.103$,

(ii) varying $h$ in the range $1.5 \leqslant h \leqslant 2.6$, and fixing $\theta=$ $0.2, s=1.25, \delta=0.005, \gamma=0.465$, (iii) varying $h$ in the range $1.5 \leqslant h \leqslant 1.57$, and fixing $\theta=$ $0.2, s=4.17, \delta=0.033, \gamma=0.31$.

For Case (i). Let $\theta=0.6, s=0.2, \delta=0.0011$, and $\gamma=0.103$, and then system (4) has only one positive fixed point $\left(x^{*}, y^{*}\right)=(0.5205,0.4220)$. After a simple calculation, we obtain $\alpha_{1}=-0.2195 \neq 0$ and $\alpha_{2}=-13.4227<0$. We can discover that system (4) with the above coefficients $(\theta, s, \delta, \gamma, h)=(0.6,0.2,0.0011,0.103,2.857) \in F_{1}$ satisfies all the conditions of the Theorem 3. Figure 1 shows the bifurcation diagram in the $h-x$ plane with the parameters given by case (i), from which we observe that the fixed point $(0.5205,0.4220)$ is stable for $h<2.857$ and loses its stability at $h=2.857$. We also observe that there is cascade of perioddoubling. The maximum Lyapunov exponents corresponding to bifurcation diagram are computed. Figure 2 shows the phase portrait of period-1, 2, 4, and 8 orbits and chaotic sets 


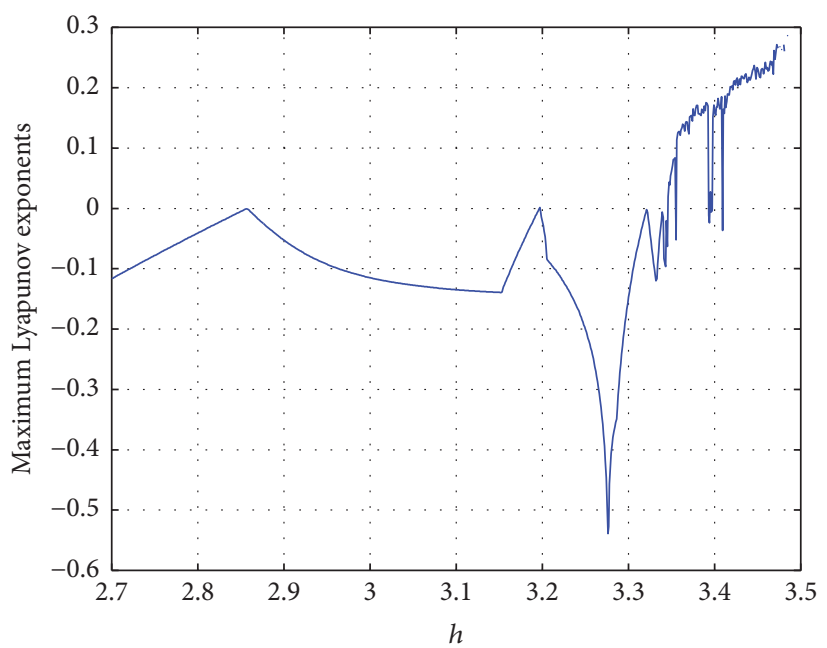

(a)

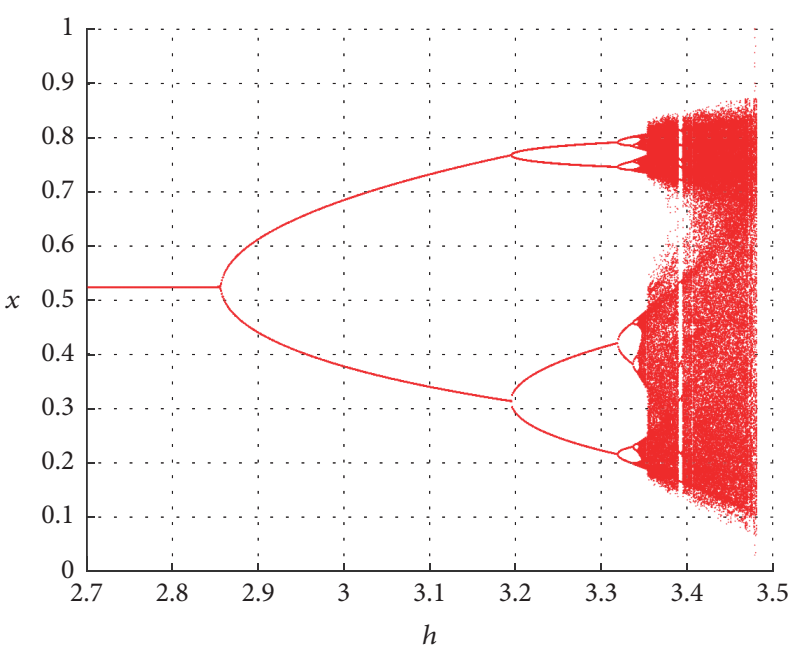

(b)

FIGURE 1: Lyapunov exponents and bifurcation diagram in the $h-x$ plane with the parameters given by case (i).

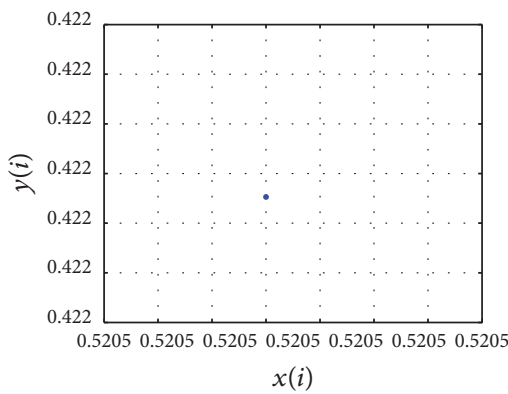

(a) $h=2.7$

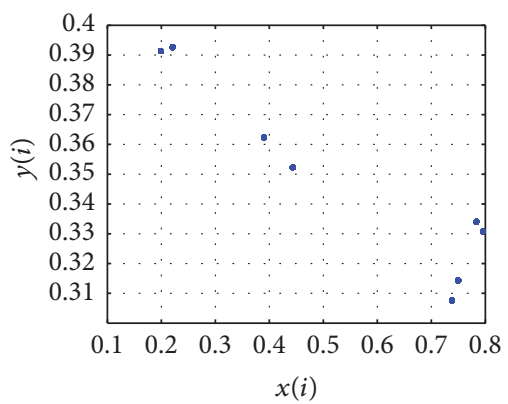

(d) $h=3.33$

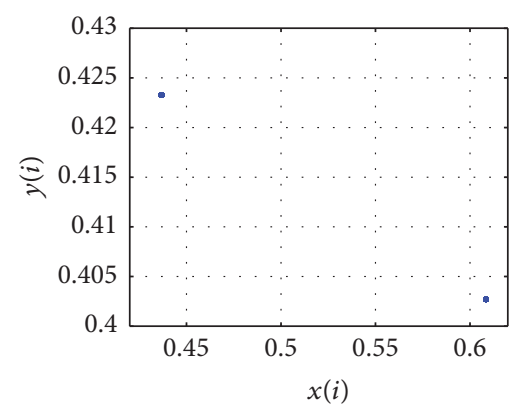

(b) $h=2.9$

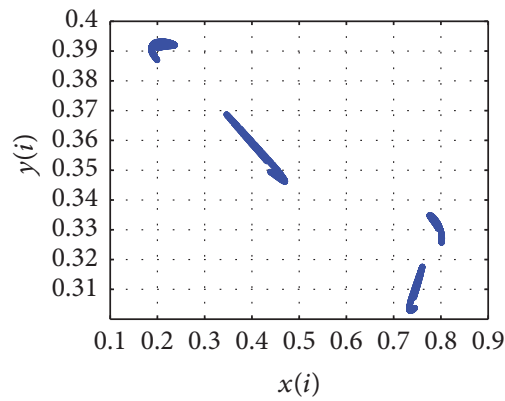

(e) $h=3.34$

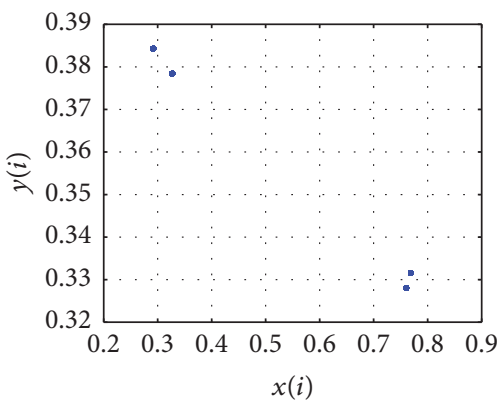

(c) $h=3.2$

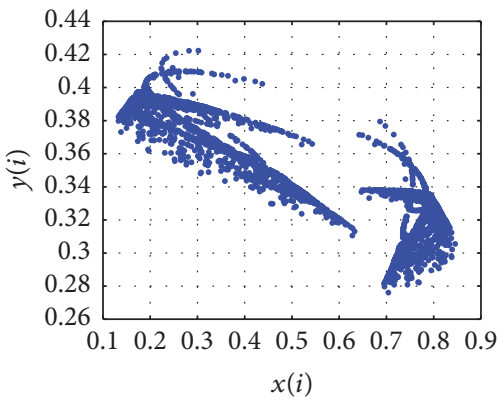

(f) $h=3.35$

FIGURE 2: Phase portraits for various values of $h$ in Figure 1.

for different values of $h$. The maximum Lyapunov exponents corresponding to $h=3.35$ are larger than 0 that confirm the existence of the chaotic sets.

For Case (ii). System (4) has only one positive fixed point $(0.376,0.2981)$ when $\theta=0.2, s=1.25, \delta=0.005$, and $\gamma=0.465$. After calculation of the positive fixed point of system (4), the Neimark-Sacker bifurcation emerges at $h=$ 1.5569 and its eigenvalues are $\lambda, \bar{\lambda}=0.6199 \pm i 0.7847$. For $h=1.5569$, we have $|\lambda, \bar{\lambda}|=1, l=0.24415>0$, $b=0.5998$. This shows the correctness of Theorem 4. From
Figure 3, we observe that the fixed point $(0.376,0.2981)$ of system (4) is stable for $h<1.5569$, that it loses its stability at $h=1.5569$, and that an invariant circle appears when the parameter $h$ exceeds 1.5569. The maximum Lyapunov exponents corresponding to Figure 3 are calculated and plotted in these. For $h>1.5669$, some Lyapunov exponents are bigger than 0 and some are smaller than 0 , so there exist stable fixed point or stable period windows in the chaotic region. In general the positive Lyapunov exponent is considered to be one of the characteristics implying the existence of chaos [37-40]. The phase portraits which are 


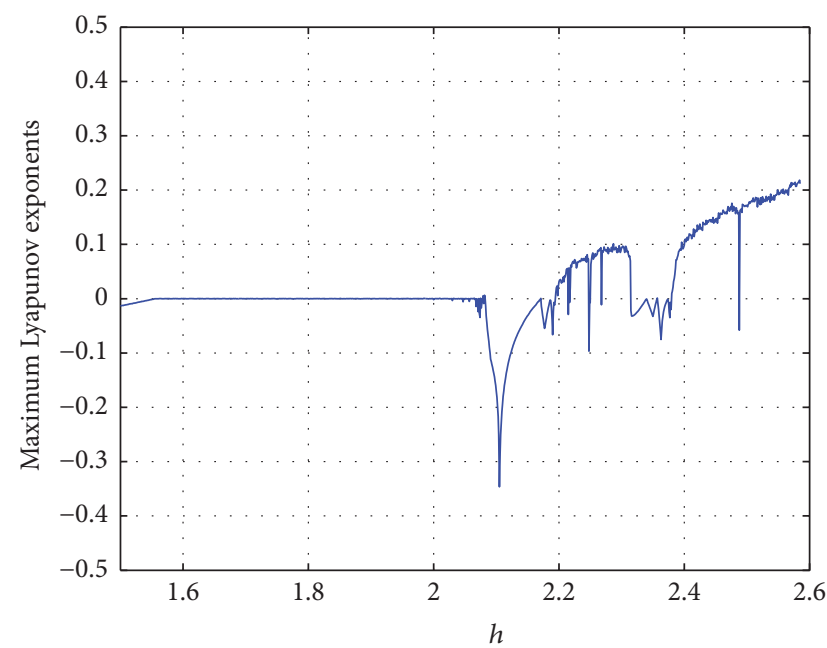

(a)

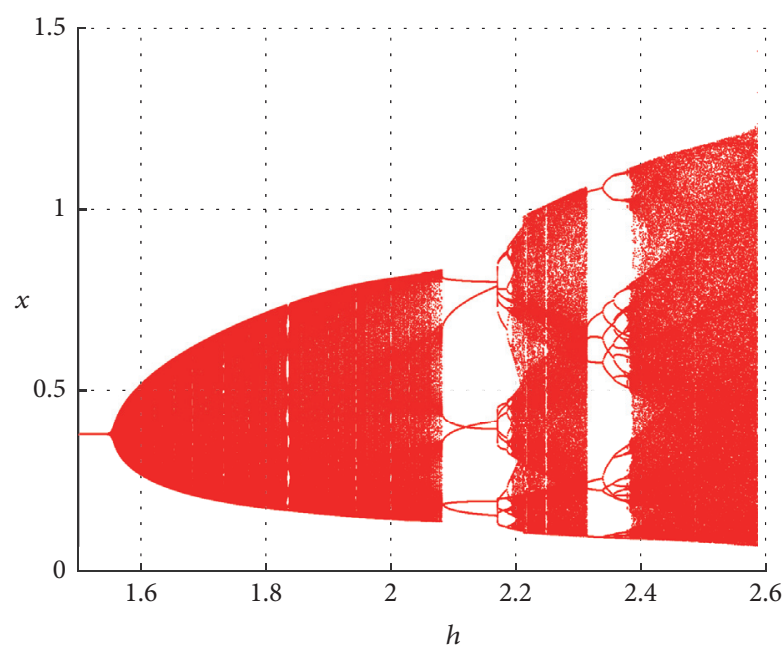

(b)

FIGURE 3: Lyapunov exponents and bifurcation diagram in the $h-x$ space for $h=1.5-2.6$ with the parameters given by case (ii).

associated with Figure 3 are displayed in Figure 4, which clearly depicts how a smooth invariant circle bifurcates from the stable fixed point $(0.376,0.2981)$ and periodic orbits and attracting chaotic sets.

For Case (iii). Vary $h$ in the range $1.5 \leqslant h \leqslant 1.57$, and fix $\theta=0.2, s=4.17, \delta=0.033$, and $\gamma=0.31$. We know that the Neimark-Sacker bifurcation emerges only from $\left(x^{*}, y^{*}\right)=(0.0823,0.2494)$ at $h=1.5107$, and its eigenvalues are $\lambda_{1,2}=-0.5945 \pm i 0.8041$. For $h=1.5107$, we have $\left|\lambda_{1,2}\right|=1, l=0.2684>0$, and $b=-3.3752$. This shows the correctness of Theorem 4. From Figure 5, we observe that the fixed point $\left(x^{*}, y^{*}\right)=(0.0823,0.2494)$ of system (4) is stable for $h<1.5107$, that it loses its stability at $h=1.5107$, and that an invariant circle appears when the parameter $h$ exceeds 1.5107. The maximum Lyapunov exponents corresponding to Figure 5 are calculated and plotted in these. Figure 6 shows phase portraits in the $s-x$ plane with the parameters given by case (iii).

\section{Chaos Control}

In this section, we apply the state feedback control method $[24,41,42]$ to stabilize chaotic orbits at an unstable fixed point of (4).

Consider the following controlled form of system (4):

$$
\begin{aligned}
F & :\left(\begin{array}{l}
x \\
y
\end{array}\right) \\
& \longrightarrow\left(\begin{array}{c}
x+h[(x+\theta y)(1-x-y)-\delta x-s x y]+u \\
y+h[s x y-(\delta+\gamma) y]
\end{array}\right)
\end{aligned}
$$

with the following feedback control law as the control force:

$$
u=-k_{1}\left(x-x^{*}\right)-k_{2}\left(y-y^{*}\right),
$$

where $k_{1}$ and $k_{2}$ are the feedback gain and $\left(x^{*}, y^{*}\right)$ is the positive point of model (4). The Jacobian matrix $J$ of the controlled system (71) and (72) evaluated at the point $\left(x^{*}, y^{*}\right)$ is given by

$$
J(x, y)=\left(\begin{array}{cc}
a_{11}-k_{1} & a_{12}-k_{2} \\
a_{21} & a_{21}
\end{array}\right),
$$

where $a_{11}, a_{12}, a_{21}, a_{22}$ are given in (26). The characteristic equation of the Jacobian matrix is

$$
\begin{gathered}
\lambda^{2}-\left(a_{11}+a_{22}-k_{1}\right) \lambda+a_{22}\left(a_{11}-k_{1}\right) \\
-a_{21}\left(a_{12}-k_{2}\right)=0
\end{gathered}
$$

Assume that the eigenvalues are given by $\lambda_{1}$ and $\lambda_{2}$; then

$$
\lambda_{1}+\lambda_{2}=a_{11}+a_{22}-k_{1}
$$

and

$$
\lambda_{1} \lambda_{2}=a_{22}\left(a_{11}-k_{1}\right)-a_{21}\left(a_{12}-k_{2}\right)
$$

The lines of marginal stability are determined by solving the equation $\lambda_{1}= \pm 1$ and $\lambda_{1} \lambda_{2}=1$. These conditions guarantee that the eigenvalues $\lambda_{1}$ and $\lambda_{2}$ have modulus less than 1.

Assume that $\lambda_{1} \lambda_{2}=1$, and from (76) we have

$$
l_{1}: k_{1} a_{22}-k_{2} a_{21}=a_{11} a_{22}-a_{12} a_{21}-1 \text {. }
$$

Assume that $\lambda_{1}=1$, and from (75) and (76) we have

$$
\begin{aligned}
l_{2}: & k_{1}\left(1-a_{22}\right)-k_{2} a_{21} \\
& =a_{11}+a_{22}-1-a_{11} a_{22}+a_{12} a_{21} .
\end{aligned}
$$

Assume that $\lambda_{1}=-1$, and from (76) we have

$$
\begin{aligned}
l_{3}: & k_{1}\left(1+a_{22}\right)-k_{2} a_{21} \\
& =a_{11}+a_{22}+1+a_{11} a_{22}-a_{12} a_{21} .
\end{aligned}
$$




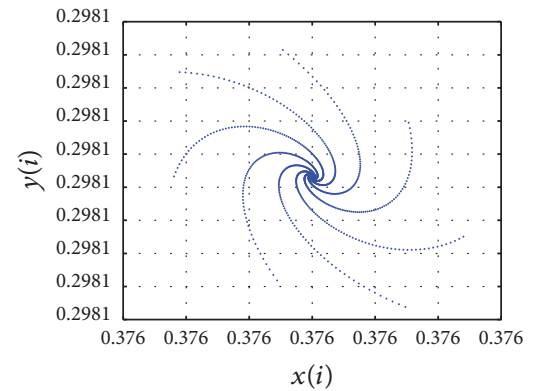

(a) $h=1.54$

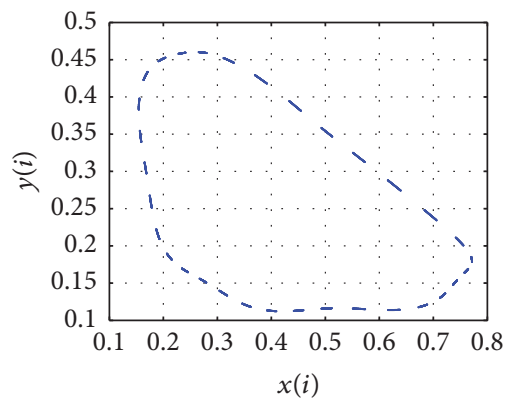

(d) $h=1.9$

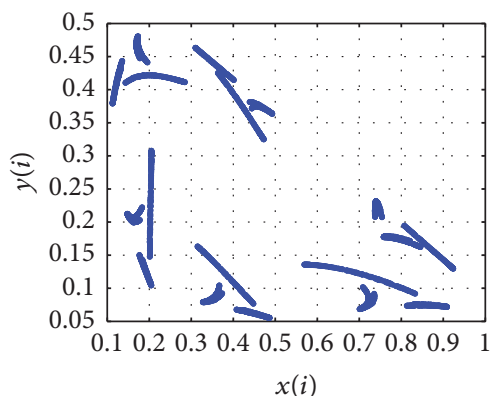

(g) $h=2.2$

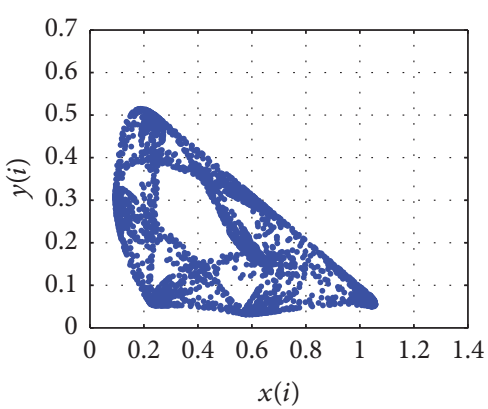

(j) $h=2.31$

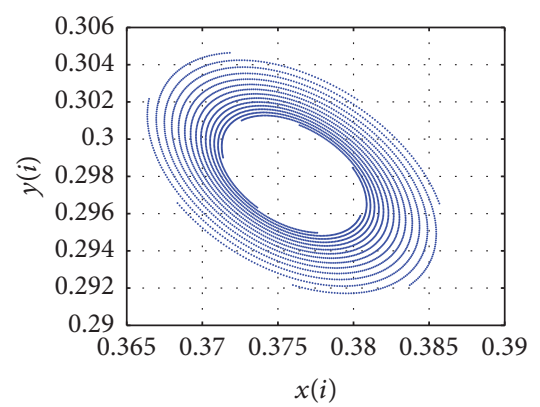

(b) $h=1.5555$

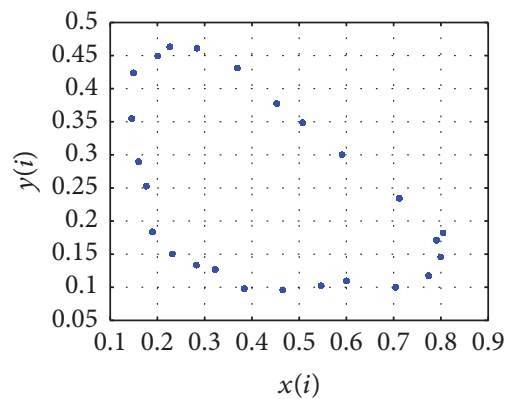

(e) $h=2$

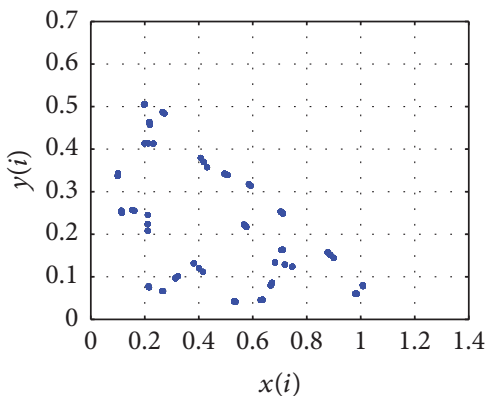

(h) $h=2.25$

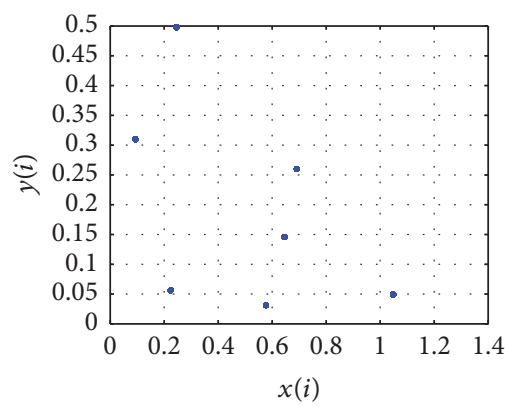

(k) $h=2.32$

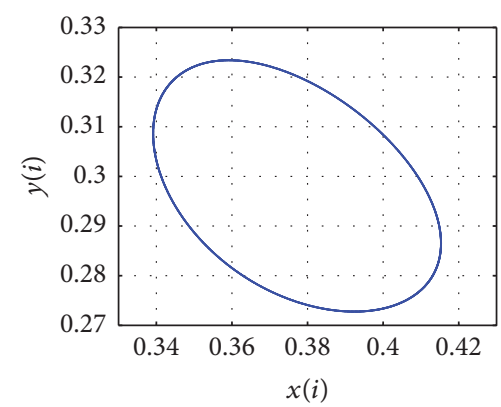

(c) $h=1.56$

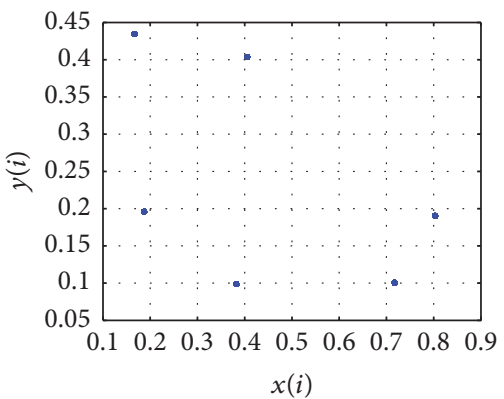

(f) $h=2.1$

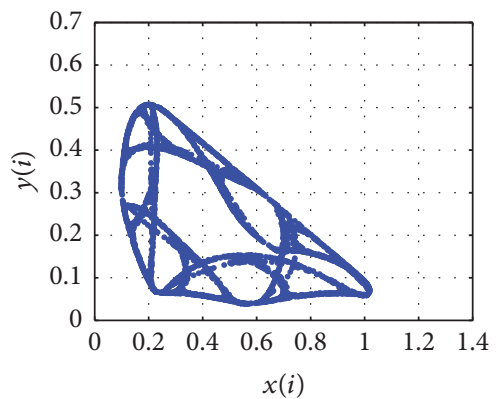

(i) $h=2.26$

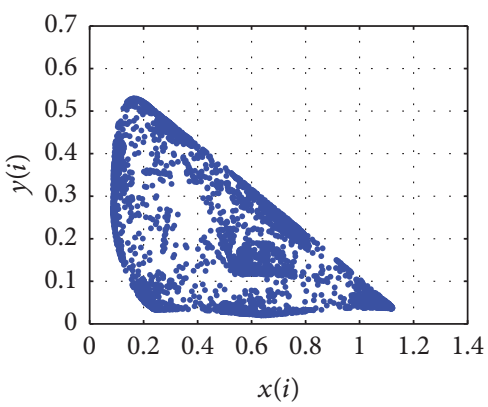

(l) $h=2.4$

Figure 4: Phase portraits for various values of $h$ in Figure 3.

The stable eigenvalues lie within a triangular region by lines $l_{1}, l_{2}$, and $l_{3}$ (see Figure 7).

We have performed some numerical simulations to see how the state feedback method controls the unstable fixed point. Parameter values are fixed as $\theta=0.2, s=1.25$, $\delta=0.005, \gamma=0.465$, and $h=2.3$. The initial value $(0.3,0.1)$, and the feedback gain $k_{1}=0.91, k_{2}=1.5$. It is shown in
Figure 8 that a chaotic trajectory is stabilized at the fixed point $\left(x^{*}, y^{*}\right)=(0.376,0.2981)$.

\section{Conclusion}

In this paper, we mainly investigated the existence and stability of the nonnegative fixed points of system (4) and 


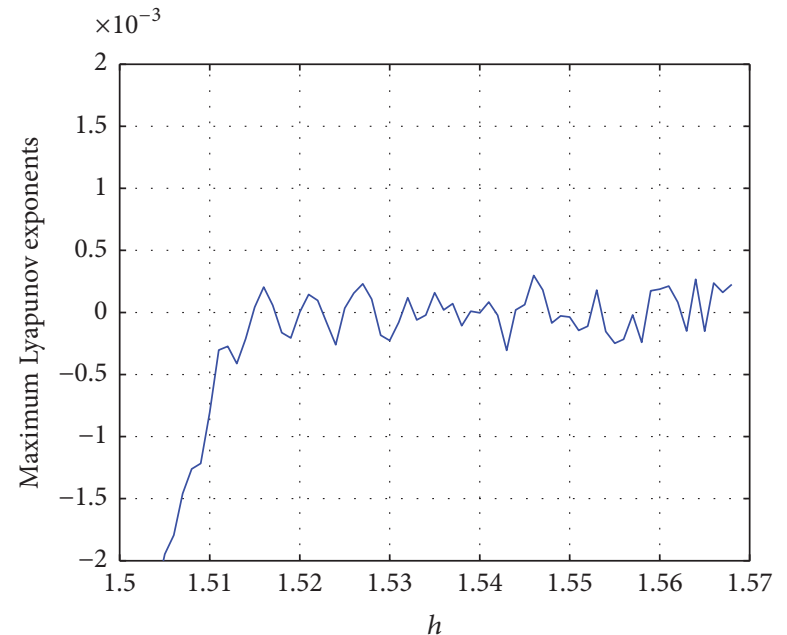

(a)

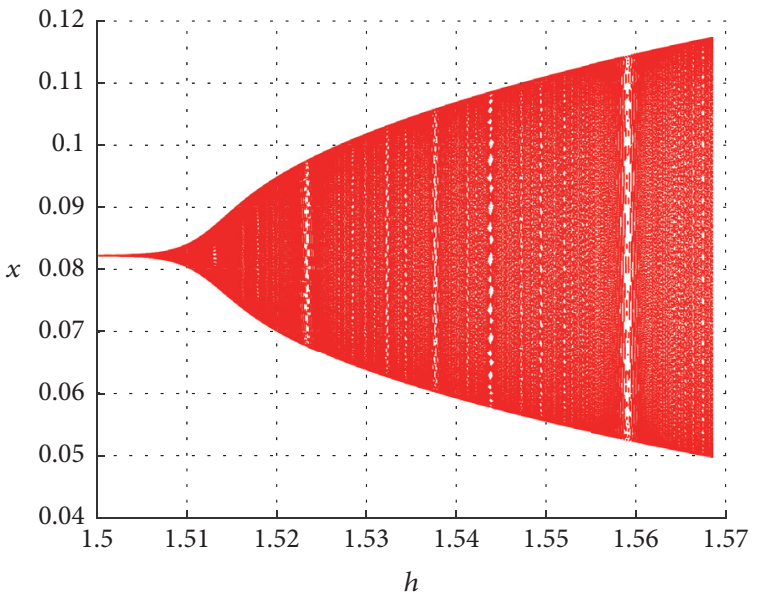

(b)

FIGURE 5: Lyapunov exponents and bifurcation diagram in the $h-x$ plane for $h=1.5-1.57$ with the parameters given by case (iii).

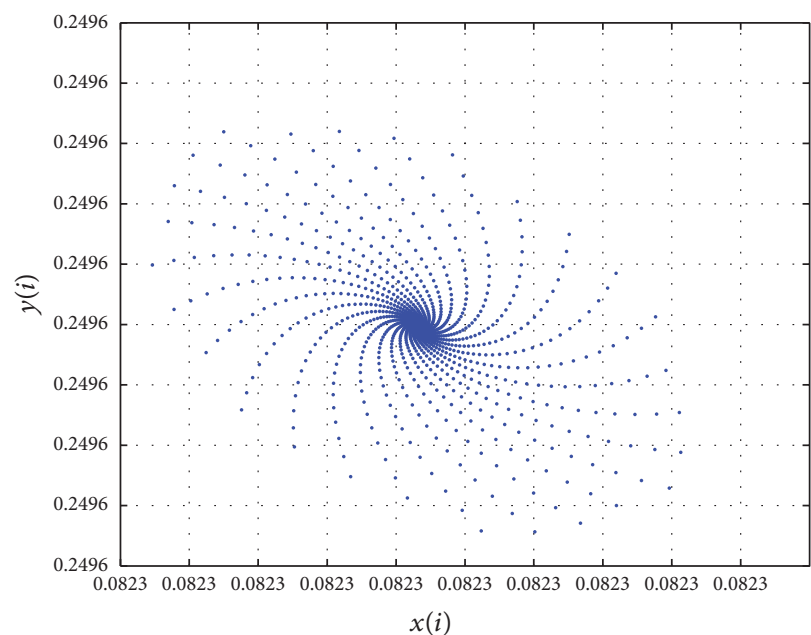

(a) $h=1.5$

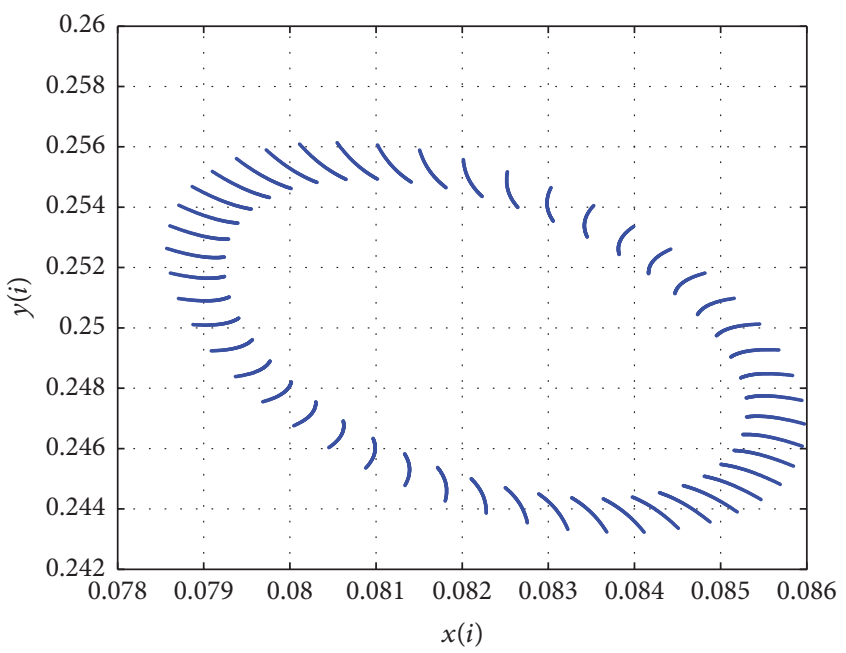

(c) $h=1.513$

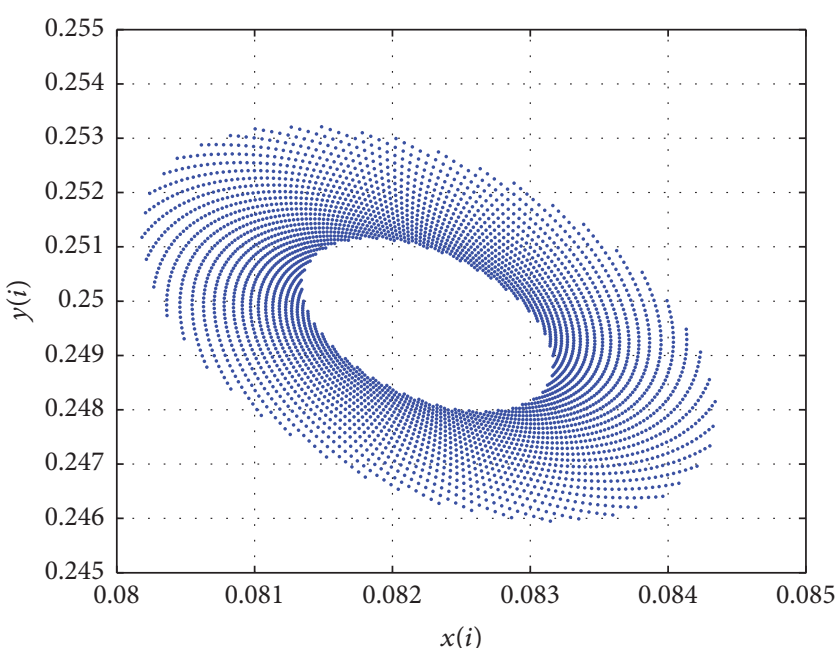

(b) $h=1.512$

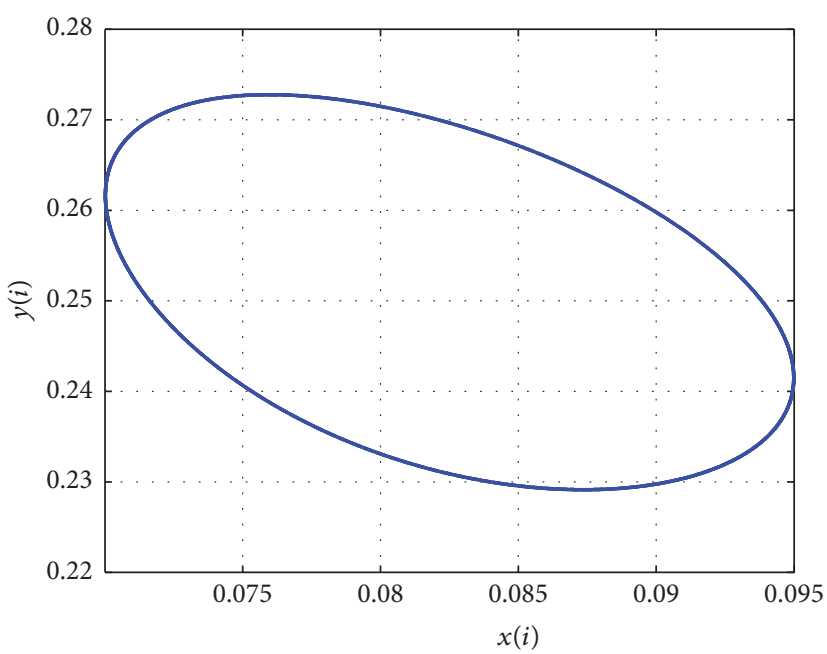

(d) $h=1.52$

Figure 6: Phase portraits for various values of $h$ in Figure 5. 


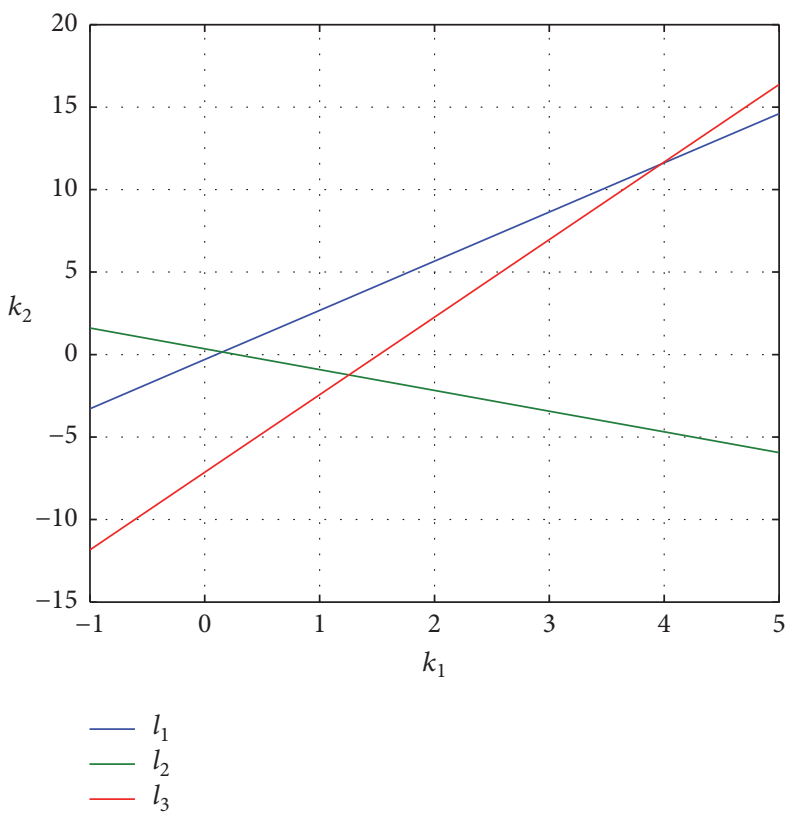

Figure 7: The bounded region for the eigenvalues of the controlled system (48), (50) in the $\left(k_{1}, k_{2}\right)$ plane for $\theta=0.2, s=1.25, \delta=0.005$, $\gamma=0.465$, and $h=2.3$.

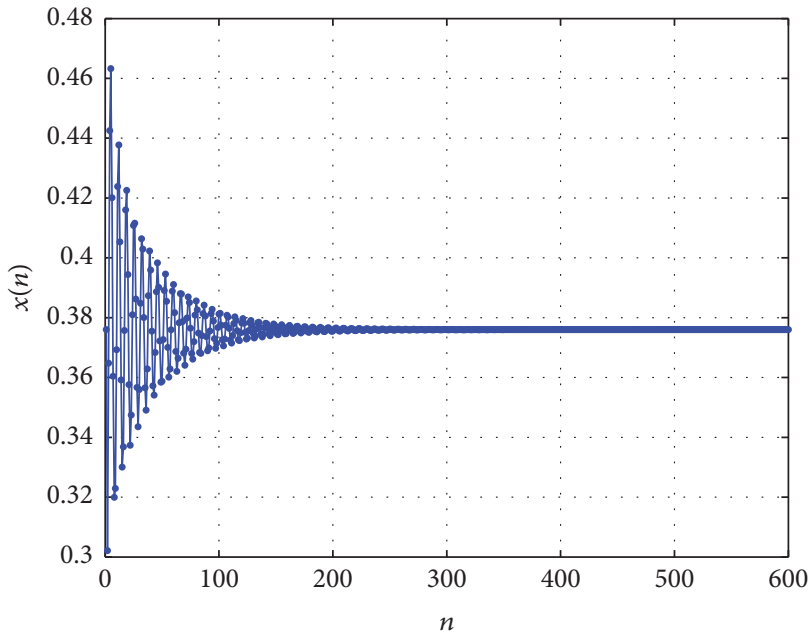

(a)

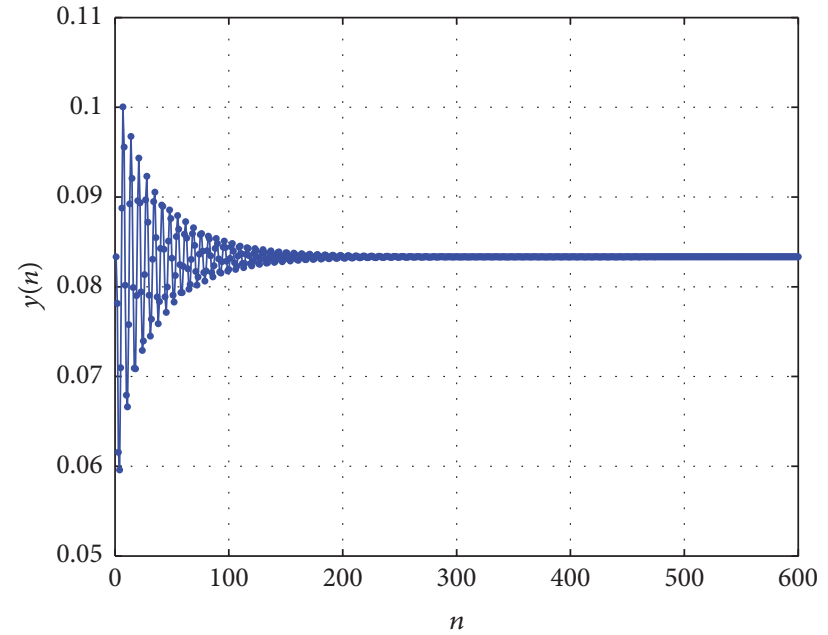

(b)

Figure 8: (a) The time responses for the state $x$ of the controlled system (48), (50) in the ( $n, x$ ) plane for $\theta=0.2, s=1.25, \delta=0.005, \gamma=0.465$, $h=2.3, k_{1}=0.91$, and $k_{2}=1.5$. The initial value is $(0.3,0.1)$. (b) The time responses for the state $y$ of the controlled system (48), (50) in the $(n, y)$ plane.

flip bifurcation and Neimark-Sacker bifurcation under some conditions at the unique positive fixed point $\left(x^{*}, y^{*}\right)$ by using manifold theorem and bifurcation theory and also proved the chaos in the sense of Marotto. Our main results are given in Propositions 1, 2 and Theorems 3, 4, and 9 and numerical simulations in Section 5. In the details of the result, when the integral step size $h$ is chosen as a bifurcation parameter, the discrete-time parasite-host system exists with much richer nonlinear dynamical behaviors. Seen from Figures 16 , there exist period-1, 2, 4, and 8 orbits, attracting invariant cycles, and even stranger chaotic attractors. These results demonstrate that the integral step size $h$ makes differences corresponding to the local and global stability of the discretetime parasite-host system. Moreover, Figure 1 shows that the system exists in flip bifurcation, which proves the correctness of Theorem 3. In addition, Figure 1 demonstrates the system exists with other complex dynamics including stable coexistence, period-doubling bifurcations. Figures 3 and 5 show that the system exists with Neimark-Sacker bifurcation, which proves the correctness of Theorem 4 . The phase portraits corresponding to Figures 1,3, and 5 are shown in Figures 2, 4, and 6. These figures show that, along with 
step $h$ increase, the stability properties of the equilibrium will be lost and the flip bifurcation, Neimark-Sacker bifurcation, and chaos phenomenon will appear. Specifically, we have stabilized the chaotic orbits at an unstable fixed point using the feedback control method.

However, in this paper we only present the numerical results. In our future work, in order to investigate the more biological significance of system (4), we expect to obtain some real data to illustrate the validity of our theoretical results and to study the effect of bifurcations and chaos on a discrete parasite-host model.

\section{Competing Interests}

The authors declare that there is no conflict of interests regarding the publication of this paper.

\section{Acknowledgments}

The project was supported by National Natural Science Foundation of He'nan Educational committee (no. 2011B110034) and Science and Technology Program of He'nan Province (no. 162102310604).

\section{References}

[1] A. Hastings and K. Higgins, "Persistence of transients in spatially structured ecological models," Science, vol. 263, no. 5150, pp. 1133-1136, 1994.

[2] A. B. T. Barbaro, K. Taylor, P. F. Trethewey, L. Youseff, and B. Birnir, "Discrete and continuous models of the dynamics of pelagic fish: application to the capelin," Mathematics and Computers in Simulation, vol. 79, no. 12, pp. 3397-3414, 2009.

[3] J. R. Beddington, C. A. Free, and J. H. Lawton, "Dynamic complexity in predator-prey models framed in difference equations," Nature, vol. 255, no. 5503, pp. 58-60, 1975.

[4] E.-G. Gu, "The nonlinear analysis on a discrete host-parasitoid model with pesticidal interference," Communications in Nonlinear Science and Numerical Simulation, vol. 14, no. 6, pp. 27202727, 2009.

[5] X. Chen, "Periodicity in a nonlinear discrete predator-prey system with state dependent delays," Nonlinear Analysis: Real World Applications, vol. 8, no. 2, pp. 435-446, 2007.

[6] N. Fang and X. X. Chen, "Permanence of a discrete multispecies Lotka-Volterra competition predator-prey system with delays," Nonlinear Analysis: Real World Applications, vol. 9, no. 5, pp. 2185-2195, 2008.

[7] Q. Fang, X. Li, and M. Cao, "Dynamics of a discrete predatorprey system with Beddington-DeAngelis function response," Applied Mathematics, vol. 3, no. 4, pp. 389-394, 2012.

[8] H.-F. Huo and W.-T. Li, "Stable periodic solution of the discrete periodic Leslie-Gower predator-prey model," Mathematical and Computer Modelling, vol. 40, no. 3-4, pp. 261-269, 2004.

[9] L. Li and Z.-J. Wang, "Global stability of periodic solutions for a discrete predator-prey system with functional response," Nonlinear Dynamics, vol. 72, no. 3, pp. 507-516, 2013.

[10] C. Lu and L. Zhang, "Permanence and global attractivity of a discrete semi-ratio dependent predator-prey system with Holling II type functional response," Journal of Applied Mathematics and Computing, vol. 33, no. 1-2, pp. 125-135, 2010.
[11] R. Abu-Saris, Z. AlSharawi, and M. H. Rhouma, "The dynamics of some discrete models with delay under the effect of constant yield harvesting," Chaos, Solitons \& Fractals, vol. 54, pp. 26-38, 2013.

[12] H. Yu, M. Zhao, S. Lv, and L. Zhu, "Dynamic complexities in a parasitoid-host-parasitoid ecological model," Chaos, Solitons and Fractals, vol. 39, no. 1, pp. 39-48, 2009.

[13] M. Zhao, H. Yu, and J. Zhu, "Effects of a population floor on the persistence of chaos in a mutual interference host-parasitoid model," Chaos, Solitons \& Fractals, vol. 42, no. 2, pp. 1245-1250, 2009.

[14] M. Zhao and L. Zhang, "Permanence and chaos in a hostparasitoid model with prolonged diapause for the host," Communications in Nonlinear Science and Numerical Simulation, vol. 14, no. 12, pp. 4197-4203, 2009.

[15] M. Zhao, L. Zhang, and J. Zhu, "Dynamics of a host-parasitoid model with prolonged diapause for parasitoid," Communications in Nonlinear Science and Numerical Simulation, vol. 16, no. 1, pp. 455-462, 2011.

[16] L. Zhu and M. Zhao, "Dynamic complexity of a host-parasitoid ecological model with the Hassell growth function for the host," Chaos, Solitons \& Fractals, vol. 39, no. 3, pp. 1259-1269, 2009.

[17] L. Cheng and H. Cao, "Bifurcation analysis of a discrete-time ratio-dependent predator-prey model with Allee effect," Communications in Nonlinear Science and Numerical Simulation, vol. 38, pp. 288-302, 2016.

[18] D. Hu and H. Cao, "Bifurcation and chaos in a discrete-time predator-prey system of Holling and Leslie type," Communications in Nonlinear Science and Numerical Simulation, vol. 22, no. 1-3, pp. 702-715, 2015.

[19] Z. Hu, Z. Teng, and H. Jiang, "Stability analysis in a class of discrete SIRS epidemic models," Nonlinear Analysis: Real World Applications, vol. 13, no. 5, pp. 2017-2033, 2012.

[20] L. Zhang, C. Zhang, and M. Zhao, "Dynamic complexities in a discrete predator-prey system with lower critical point for the prey," Mathematics and Computers in Simulation, vol. 105, pp. 119-131, 2014.

[21] X. Liu and D. Xiao, "Complex dynamic behaviors of a discretetime predator-prey system," Chaos, Solitons \& Fractals, vol. 32, no. 1, pp. 80-94, 2007.

[22] H. N. Agiza, E. M. ELabbasy, H. EL-Metwally, and A. A. Elsadany, "Chaotic dynamics of a discrete prey-predator model with Holling type II," Nonlinear Analysis: Real World Applications, vol. 10, no. 1, pp. 116-129, 2009.

[23] F. Chen, "Permanence and global attractivity of a discrete multispecies Lotka-Volterra competition predator-prey systems," Applied Mathematics and Computation, vol. 182, no. 1, pp. 3-12, 2006.

[24] Z. He and X. Lai, "Bifurcation and chaotic behavior of a discretetime predator-prey system," Nonlinear Analysis: Real World Applications, vol. 12, no. 1, pp. 403-417, 2011.

[25] E. M. Elabbasy, H. N. Agiza, H. El-Metwally, and A. A. Elsadany, "Bifurcation analysis, chaos and control in the Burgers mapping," International Journal of Nonlinear Science, vol. 4, no. 3, pp. 171-185, 2007.

[26] E. M. Elabbasy, A. A. Elsadany, and Y. Zhang, "Bifurcation analysis and chaos in a discrete reduced Lorenz system," Applied Mathematics and Computation, vol. 228, pp. 184-194, 2014.

[27] H. Shu and J. Wei, "Bifurcation analysis in a discrete BAM network model with delays," Journal of Difference Equations and Applications, vol. 17, no. 1, pp. 69-84, 2011. 
[28] D. Fan and J. Wei, "Bifurcation analysis of discrete survival red blood cells model," Communications in Nonlinear Science and Numerical Simulation, vol. 14, no. 8, pp. 3358-3368, 2009.

[29] Z. Jing, Y. Chang, and B. Guo, "Bifurcation and chaos in discrete FitzHugh-Nagumo system," Chaos, Solitons \& Fractals, vol. 21, no. 3, pp. 701-720, 2004.

[30] D. Ebert, M. Lipsitch, and K. L. Mangin, “The effect of parasites on host population density and extinction: experimental epidemiology with Daphnia and six microparasites," The American Naturalist, vol. 156, no. 5, pp. 459-477, 2000.

[31] S. B. Hsu and T. W. Huang, "Global stability for a class of predator-prey systems," SIAM Journal on Applied Mathematics, vol. 55, no. 3, pp. 763-783, 1995.

[32] F. R. Marotto, "Snap-back repellers imply chaos in Rn," Journal of Mathematical Analysis and Applications, vol. 63, no. 1, pp. 199223, 1978.

[33] J. Carr, Application of Center Manifold Theory, Springer, New York, NY, USA, 1981.

[34] J. Guckenheimer and P. Holmes, Dynamical Systems and Bifurcation of Vector Fields, Springer, New York, NY, USA, 1983.

[35] Y. A. Kuznetsov, Elements of applied bifurcation theory, vol. 112 of Applied Mathematical Sciences, Springer, New York, USA, Second edition, 1998.

[36] S. Wiggins, Introduction to Applied Nonlinear Dynamical Systems and Chaos, Springer, New York, NY, USA, 1990.

[37] K. T. Alligood, K. T. Sauer, and J. A. Yorke, Chaos-An Introduction to Dynamical Systems, Springer, New York, NY, USA, 1996.

[38] E. Ott, Chaos in dynamical systems, Cambridge University Press, Cambridge, Mass, USA, Second edition, 2002.

[39] S. N. Elaydi, An Introduction to Difference Equations, Springer, New York, NY, USA, 2005.

[40] S. Lynch, Dynamical Systems with Applications Using Mathematica, Birkhäuser, Boston, Mass, USA, 2007.

[41] G. Chen and X. Dong, From Chaos to Order: Perspectives, Methodologies, and Applications, World Scientific, Singapore, 1998.

[42] S. Elaydi, An introduction to difference equations, Undergraduate Texts in Mathematics, Springer, New York, USA, Third edition, 2005. 


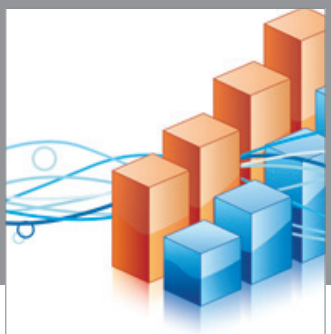

Advances in

Operations Research

vatem alat4

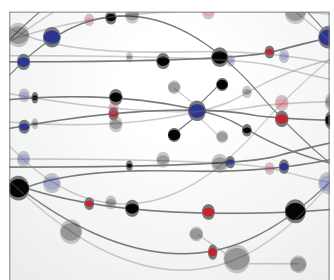

\section{The Scientific} World Journal
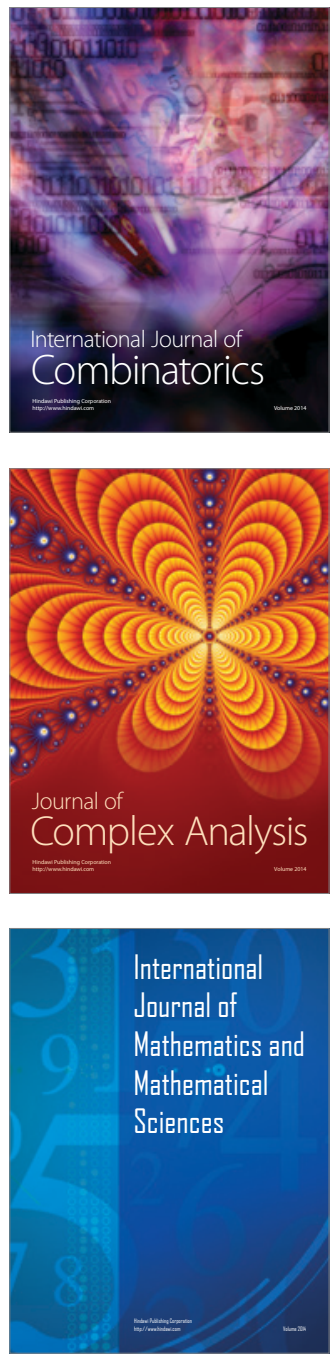
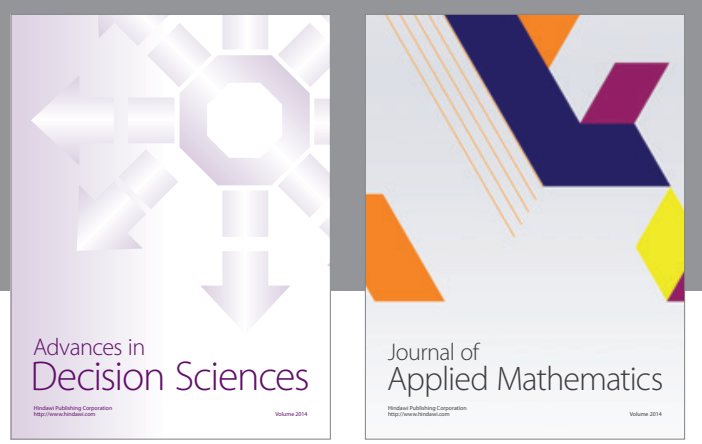

Algebra

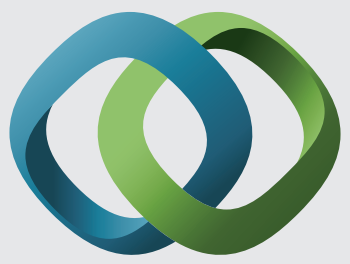

\section{Hindawi}

Submit your manuscripts at

https://www.hindawi.com
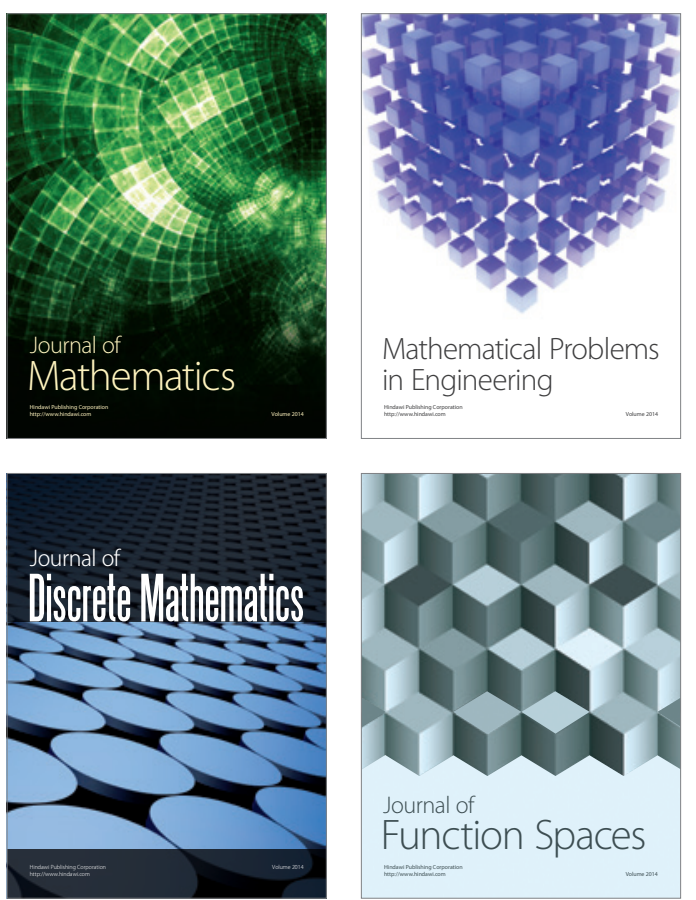

Mathematical Problems in Engineering
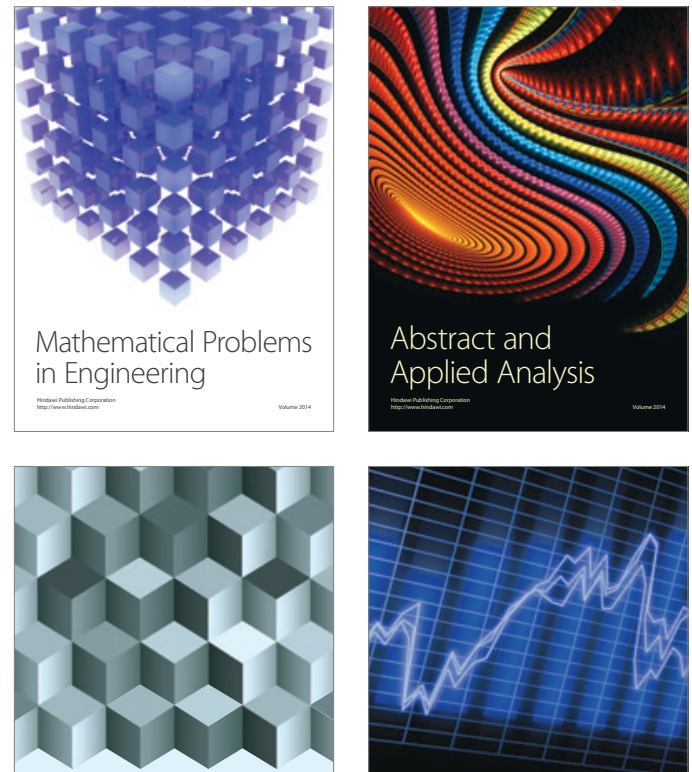

Journal of

Function Spaces

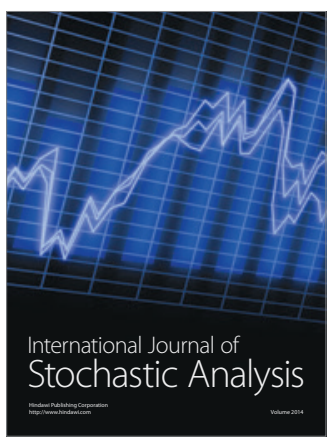

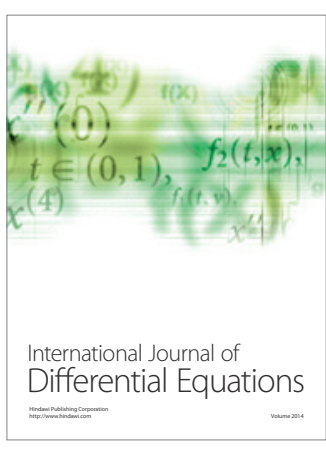
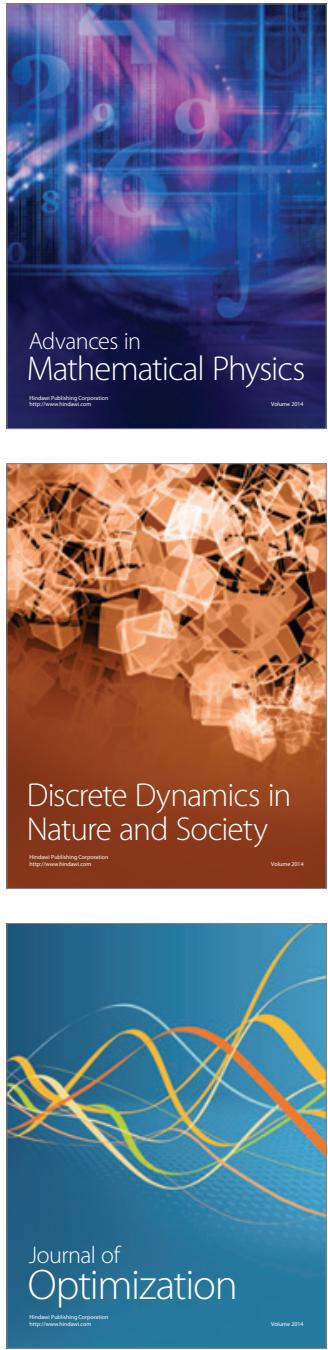